\title{
The Probabilistic Treatment of Phase Separations in Lattice Models Composed of More Than Two Types of Particles
}

By

\author{
Koji KURODA*
}

\section{Introduction}

The probabilistic treatment of phase separation for two-dimensional Ising model was made by Minlos and Sinai [1], [2]. They showed the existence of a lump of minus spins with the shape of nearly a square with the breadth of about $N^{1 / 2}$ asymptotically with probability one when the number of minus spins $N$ tends to infinity.

In this paper we consider the system in $\mathbb{Z}^{2}$ composed of more than two types of particles and show by refining the method of Minlos and Sinai the occurrence of phase separations of various types.

In particular, when the system is composed of three types of particles, the following two types of phase separations called type I and II are typical; the first one is "there exist two disjoint square lumps occupied by $B$ - and $C$-particles respectively in the 'sea' of $A$-particles", and the second one is "there exists one square lump consisting of 'core-part' and 'shell-part' in the 'sea' of $A$-particles, its 'core-part' is occupied by $C$-particles being also a spuare, and its 'shell-part' is occupied by $B$-particles", (See Fig. 1).

In Ising model we obtain the various estimates of correlation functions by using the simple symmetry of plus and minus spins. But this system does not have such simple symmetry. So, we consider the system composed of four types of particles, in which the symmetry is satisfied, and show the occurrence of phase separations of type I and II.

In Section 1 we describe the model precisely and give the definitions which

Communicated by H. Araki, February 23, 1981. Revised May 20, 1981.

* Department of Mathematics, Tsukuba University, Ibaraki 305, Japan.

Present address: Department of Mathematics, Keio University, Hiyoshi, Kanagawa 223, Japan. 

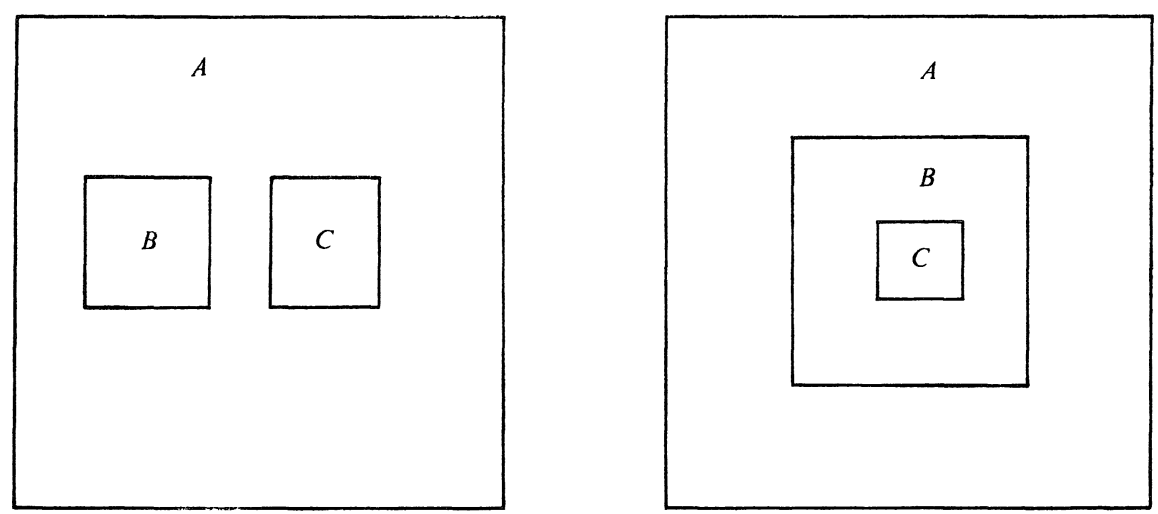

Figure 1.

will be used in the sequel. We state our results rigorously in Section 2. Several properties of correlation functions of contours are stated in Sections 3 and 4. In Section 5 we estimate the expectation value and the variance of number of particles. We give the proof of our theorems in Sections 6 and 7 .

\section{$\S 1$. The Description of the Model}

In this section we describe the model and give the definitions which will be used in the sequel.

We consider the interaction system on $\boldsymbol{Z}^{2}$ which is composed of $n(>2)$ types of particles, denoted by $A, B, \ldots$, and $N$.

In Ising model each configuration in a bounded region $V$ under the pure boundary condition is represented by the family of closed lines drawn between the different spins. While, such lines may branch in multi-component system composed of more than two types of particles.

In this paper we consider the model in which configurations including such branching lines are not allowed. Therefore, in our model only the configurations as in the Figure 2 are allowed. When the number of types of particles is more than two the configuration cannot be represented by the family of closed lines. So, we introduce the new notion of "contours". We call the triple $\bar{\Gamma}=(\Gamma, a, b)$ contour, where $a$ and $b$ are the types of the particles being contact with the closed line $\Gamma$ from outside and inside. When the configuration in $V^{c}$ is fixed to $\omega_{A}$, each configuration in $V$ is represented by the family of contours $\left(\bar{\Gamma}_{1}, \ldots, \bar{\Gamma}_{s}\right)$, where $\omega_{A}$ is the configuration given by 


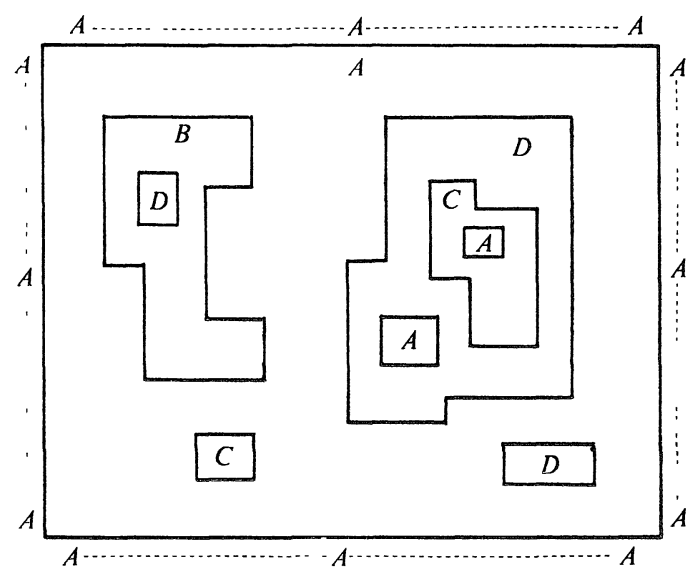

Figure 2.

$$
\omega_{A}(t)=A \quad \text { for all } \quad t \in \mathbb{Z}^{2} .
$$

When the configuration in $V$ is represented by $\left(\bar{\Gamma}_{1}, \ldots, \bar{\Gamma}_{s}\right)$ we associate to them "potential energy"

$$
U_{V}\left(\bar{\Gamma}_{1}, \ldots, \bar{\Gamma}_{s}\right)=\sum_{i=1}^{s} E\left(\bar{\Gamma}_{i}\right),
$$

where $E(\cdot)$ is the real valued function defined on the set of all contours. Further we assume that $E(\cdot)$ has the formula given by

$$
E(\bar{\Gamma})=\varepsilon(a, b) \widetilde{E}(\Gamma) \quad(\bar{\Gamma}=(\Gamma, a, b))
$$

where $\widetilde{E}(\cdot)$ is the function defined on the set of all closed lines in $\mathbb{Z}^{2}$ and $\varepsilon(a, b)$ is the positive number determined by the set $\{a, b\}$. We assume the further conditions (c.1) and (c.2) on $\widetilde{E}(\cdot)$ and $\{\varepsilon(a, b)\}_{a, b \in S}$, where $S=\{A, B, \ldots, N\}$.

$$
|\widetilde{E}(\Gamma)-| \Gamma||<E_{0}(|\Gamma|),
$$

where $E_{0}(\cdot)$ is the function defined on $[0, \infty)$ satisfying

$$
0<E_{0}(k)<k / 4, \quad E_{0}(k)=o(k) .
$$

(c.2) For each $a$ and $b(a \neq b)$ there exists a permutation in $S f=f(a, b)$ satisfying 1) $f(a)=b$ and 2) $\varepsilon(f(c), f(d))=\varepsilon(c, d)$ for all $c$ and $d \in S$.

We prepare some terminologies which will be used in the sequel. Let $d\left(x, y^{\prime}\right)$ be the distance between $x=\left(x_{1}, x_{2}\right)$ and $y=\left(y_{1}, y_{2}\right)$ given by

$$
d(x, y)=\left|x_{1}-y_{1}\right|+\left|x_{2}-y_{2}\right| .
$$

We say two sites $x$ and $y$ are adjacent if $d(x, y)=1$. For a given bounded 
$V \subset \mathbb{Z}^{2}$, we denote the set of all sites in $\mathbb{Z}^{2} \backslash V$ adjacent to some site in $V$ by $\partial V$ and the set of all sites in $V$ adjacent to some site in $\mathbb{Z}^{2} \backslash V$ by $\partial_{\text {in }} V$. Further we denote the set of sites enclosed by $\Gamma$ by $v(\Gamma)$ and put $\theta(\Gamma)=v(\Gamma) \backslash \partial_{\text {in }} v(\Gamma), w(\Gamma)$ $=v(\Gamma) \cup \partial v(\Gamma)$. Let $\bar{V}$ be the region surrounded by the outer boundary of $V$. The region $\bar{V} \backslash V$ is called inner part of $V$. If $V$ is simply connected, then the inner part of $V$ is vacant. We say the family of contours $\left(\bar{\Gamma}_{1}, \ldots, \bar{\Gamma}_{s}\right)$ compatible with $(V, A)$ if each $\Gamma_{i}$ does not surround the inner part of $V$ and there exists a configuration under which the totality of contours is given by $\left(\bar{\Gamma}_{1}, \ldots, \bar{\Gamma}_{s}\right)$. We denote the set of all families of contours $\xi=\left(\bar{\Gamma}_{1}, \ldots, \bar{\Gamma}_{s}\right)$ compatible with $(V, A)$ by $\Omega_{V, A}$. We also say the family of contours $\left(\bar{\Gamma}_{1}, \ldots, \bar{\Gamma}_{s}\right)$ admissible with $(V, A)$ if there exists a configuration $\xi \in \Omega_{V, A}$ satisfying $\left\{\bar{\Gamma}_{1}, \ldots, \bar{\Gamma}_{s}\right\} \subset \xi$. When $\bar{\Gamma} \in \xi$ is not surrounded by any other contours in $\zeta$ we call $\bar{\Gamma}$ outer and denote the totality of outer contours in $\xi$ by $\xi_{\text {out }}$. For simplicity we denote the outer and inner condition of $\bar{\Gamma}$ by $o(\bar{\Gamma})$ and $i(\bar{\Gamma})$ respectively.

Finally we define the Gibbs measure $P_{V, A}(\cdot)$ on $\Omega_{V, A}$ by

$$
P_{V, A}(\xi)=\frac{1}{Z_{V, A}} \exp \left(-\beta U_{V, A}(\xi)\right),
$$

where $\beta=1 / k T$ ( $k$ : Boltzmann's constant, $T$ : absolute temperature).

\section{§2. Main Results}

In this section we restrict our attention to the model composed of only four types of particles, denoted by $A, B, C$, and $D$, and give the interaction parameters $\{\varepsilon(a, b)\}_{a, b}$ by

$$
\begin{aligned}
\varepsilon(A, B) & =\varepsilon(B, C)=\varepsilon(C, D)=\varepsilon(D, A) \\
& =\varepsilon_{1} \ll \varepsilon_{2}=\varepsilon(A, C)=\varepsilon(B, D) .
\end{aligned}
$$

It is easily seen that $\{\varepsilon(a, b)\}$ given by (3.1) satisfies the condition (C.2). (In $\S 3$ and $\S 4$ we prove the various properties of correlation functions in models satisfying (C.1) and (C.2).)

We call a contour $\bar{\Gamma} c_{0}$-large if $|\Gamma|>c_{0} \ln |V|$. Other contours are called $c_{0}$-small. We also call the $c_{0}$-large contour which is not surrounded by any other $c_{0}$-small contours "phase boundary". By these boundaries $V$ is divided into several regions. When the type of particles in the inner boundary of the region is $A$, we call it " $A$-phase". The totality of " $A$-phase" is denoted by $\theta_{A}$. Similarly $B-, C$-, and $D$-phases are defined. We also denote the greatest 
connected component of $\theta_{a}$ by $\theta_{a}^{\max }$, and denote the longest boundary between $a$-type particles and $b$-type particles by $\Gamma_{a, b}^{\max }$.

Before describing our results we define the conditional Gibbs measures as follows. Put

$$
\begin{array}{r}
N_{V}^{(1)}=\left\{\xi \in \Omega_{V, A} ;\left|N_{B}(\xi ; V)-r_{B}\right| V||<\rho_{1}^{*}|V|\right. \\
\text { and } \left.\left|N_{D}(\xi: V)-r_{D}\right| V||<\rho_{1}^{*}|V|\right\}
\end{array}
$$

and

$$
\begin{aligned}
N_{V}^{(2)}=\left\{\xi \in \Omega_{V, A} ; \mid N_{B}(\xi ; V)\right. & -r_{B}|V|\left|<\rho_{1}^{*}\right| V \mid \\
& \text { and } \left.\left|N_{C}(\xi: V)-r_{C}\right| V||<\rho_{1}^{*}|V|\right\}
\end{aligned}
$$

where $\rho_{1}^{*}$ is the function of $\beta$ satisfying $\rho_{1}^{*}(\beta) / \exp \left(-3 \beta \varepsilon_{1}\right) \downarrow 0$ as $\beta \rightarrow \infty$. and its precise definition is given in Section 3. We define the conditional Gibbs measures $P_{N_{V}^{(1)}}$ and $P_{N_{V}^{(2)}}$ by

$$
\begin{aligned}
& P_{N_{V}^{(1)}}(\cdot)=P_{V, A}\left(\cdot \mid N_{V}^{(1)}\right) \\
& P_{N_{V}^{(2)}}(\cdot)=P_{V, A}\left(\cdot \mid N_{V}^{(2)}\right)
\end{aligned}
$$

Let $\left\{E_{V} \subset \Omega_{V, A}\right\}$ be the sequence of events. We say $\left\{E_{V}\right\}$ occurs asymptotically with $P_{N_{V}^{(i)}}$-probability 1 if

$$
P_{V, A}\left(E_{V}\right) \longrightarrow 1 \text { as }|V| \longrightarrow \infty
$$

and denote it by

$$
E_{V} \quad \text { a.w. } P_{N_{V}^{(i)}} \text {-prob. } 1 \text {. }
$$

We state our results in the following two theorems.

Theorem 2.1. For sufficiently large $\beta$, the following 1)-5) are satisfied asymptotically with $P_{N_{V}^{(1)}}$-prob. 1 ;

1) ||$\theta_{A}\left|-\left(1-r_{B}-r_{D}\right)\right| V||<e^{-3 \beta \varepsilon_{1}}|V|$

||$\theta_{B}\left|-r_{B}\right| V||<e^{-3 \beta \varepsilon_{1}}|V|$

||$\theta_{D}\left|-r_{D}\right| V||<e^{-3 \beta \varepsilon_{1}}|V|$

2) $\left|N_{a}\left(\theta_{a}\right)-\rho_{1}^{* *}\right| \theta_{a}||<e^{-(3 / 2) \beta \varepsilon_{1}}|V|^{3 / 4} \quad\left(a=A, B, D, \rho_{1}^{* *}=1-\rho_{1}^{*}\right)$

3) $\quad|| \theta_{B}^{\max }\left|-r_{B}\right| V||<\beta^{-\alpha}|V|$

||$\theta_{D}^{\max }\left|-r_{D}\right| V||<\beta^{-\alpha}|V|$

for each $\alpha$ satisfying $0<\alpha<2$,

4) $\left.\left.|| \partial \theta_{B}^{\max }\left|-4 r_{B}^{1 / 2}\right| V\right|^{1 / 2}\left|<2 k_{0}(\beta)\right| V\right|^{1 / 2}$

$\left.\left.|| \partial \theta_{D}^{\max }\left|-4 r_{D}^{1 / 2}\right| V\right|^{1 / 2}\left|<2 k_{0}(\beta)\right| V\right|^{1 / 2}$ 
5) $\theta_{B}^{\max } \cap \theta\left(\partial \theta_{D}^{\max }\right)=\varnothing$

where $k_{0}(\beta)=\left(8 u_{0}+1\right) /\left(\beta \varepsilon_{1}-u_{0}\right), \quad u_{0}=\ln 6+2 / c_{0}, \quad c_{0}<1 / 2 \ln 3$, and $N_{a}\left(\theta_{a}\right)$ is the number of a-particles in $0_{a}$.

Theorem 2.2. For sufficiently large $\beta$ the following 1)-5) are satisfied asymptotically with $P_{N_{V}^{(2)}}$-prob. 1 ;

1) ||$\theta_{A}\left|-\left(1-r_{B}-r_{C}\right)\right| V||<e^{-3 \beta c_{1}}|V|$

||$\theta_{B}\left|-r_{B}\right| V||<e^{-3 \beta \varepsilon_{1}}|V|$

||$\theta_{C}\left|-r_{C}\right| V||<e^{-3 \beta \varepsilon_{1}}|V|$,

2) $\left|N_{a}\left(\theta_{a}\right)-\rho_{1}^{* *}\right| \theta_{a}||<e^{-(3 / 2) \beta \varepsilon_{1}}|V|^{3 / 4} \quad(a=A, B, C)$,

3) ||$\theta_{B}^{\max }\left|-r_{B}\right| V||<\beta^{-\alpha}|V|$

||$\theta_{C}^{\max }\left|-r_{C}\right| V||<\beta^{-\alpha}|V| \quad$ for each $0<\alpha<2$,

4) $\left.|| \Gamma_{A, B}^{\max }\left|-4\left(r_{B}+r_{C}\right)^{1 / 2}\right| V\right|^{1 / 2}<2 k_{0}(\beta)|V|^{1 / 2}$,

5) $\left.|| \Gamma_{B, C}^{\max }\left|-4 r_{C}^{1 / 2}\right| V\right|^{1 / 2}<2 k_{0}(\beta)|V|^{1 / 2}$,

6) $\Gamma_{B, C}^{\max } \subset \theta\left(\Gamma_{A, B}^{\max }\right)$.

\section{§3. Properties of Correlation Functions}

In this section we treat the system satisfying the conditions (c.1) and (c.2) in Section 1.

For a given admissible family of contours $\left(\bar{\Gamma}_{1}, \ldots, \bar{\Gamma}_{s}\right)$ in $V$, the correlation function $\tau_{V, A}\left(\bar{\Gamma}_{1}, \ldots, \bar{\Gamma}_{s}\right)$ is defined by

$$
\tau_{V, A}\left(\bar{\Gamma}_{1}, \ldots, \bar{\Gamma}_{s}\right)=P_{V, A}\left(\mathscr{B}\left(\bar{\Gamma}_{1}, \ldots, \bar{\Gamma}_{s}\right)\right),
$$

where $\mathscr{B}\left(\bar{\Gamma}_{1}, \ldots, \bar{\Gamma}_{s}\right)=\left\{\xi \in \Omega_{V, A} ; \bar{\Gamma}_{1}, \ldots, \bar{\Gamma}_{s} \in \xi\right\}$.

We say the family of contours whose outer conditions are $A$ in $V\left(\bar{\Gamma}_{1}, \ldots, \bar{\Gamma}_{s}\right)$ "mutually disjoint" if there exists at least one configuration $\zeta \in \Omega_{V, A}$ such that $\vec{\Gamma}_{1}, \ldots, \bar{\Gamma}_{s} \in \xi_{\text {out }}$. We also say the family of contours in $\boldsymbol{Z}^{2}$ "mutually disjoint" if it is "mutually disjoint" in $V$ for some large $V$.

For a mutually disjoint family of contours of type $A\left(\bar{\Gamma}_{1}, \ldots, \bar{\Gamma}_{s}\right)$ the correlation function of outer contours $\rho_{V, A}\left(\bar{\Gamma}_{1}, \ldots, \bar{\Gamma}_{s}\right)$ is defined by

$$
\rho_{V, A}\left(\bar{\Gamma}_{1}, \ldots, \bar{\Gamma}_{s}\right)=P_{V, A}\left(C\left(\bar{\Gamma}_{1}, \ldots, \bar{\Gamma}_{s}\right)\right)
$$

where $C\left(\bar{\Gamma}_{1}, \ldots, \bar{\Gamma}_{s}\right)=\left\{\xi \in \Omega_{V . A} ; \bar{\Gamma}_{1}, \ldots, \bar{\Gamma}_{s} \in \xi_{\text {out }}\right\}$. 
These correlation functions satisfy the chain of equations as follows,

$$
\left\{\begin{array}{l}
\rho_{V, A}\left(\bar{\Gamma}_{1}, \ldots, \bar{\Gamma}_{s}\right)=\exp \left(-\beta E\left(\bar{\Gamma}_{1}\right)\right)\left\{\rho_{V, A}\left(\bar{\Gamma}_{2}, \ldots, \bar{\Gamma}_{s}\right)\right. \\
\quad+\sum_{h=1}^{\alpha} \frac{(-1)^{k}}{k !} \sum_{V, k}^{(1)} \rho_{V, A}\left(\bar{\Gamma}_{2}, \ldots, \bar{\Gamma}_{s}, \bar{\Delta}_{1}, \ldots, \bar{\Delta}_{h}\right) \\
\left.-\sum_{V}^{(2)} \rho_{V, A}\left(\bar{\Gamma}_{2}, \ldots, \bar{\Gamma}_{s}, \bar{\Lambda}\right)\right\} \quad \text { if } s>1 \\
\rho_{V, A}\left(\bar{\Gamma}_{1}\right)=\exp \left(-\beta E\left(\bar{\Gamma}_{1}\right)\right)\left\{1+\sum_{h=1}^{\alpha} \frac{(-1)^{h}}{k !} \sum_{V, h}^{(1)} \rho_{V, A}\left(\bar{\Delta}_{1}, \ldots, \bar{\Delta}_{h}\right)\right. \\
\left.-\sum_{V^{\prime}}^{(2)} \rho_{V, A}(\bar{\Lambda})\right\} \quad \text { if } s=1
\end{array}\right.
$$

where the sum $\sum_{V, h}^{(1)}$ takes over all the $k$-ordered pairs of contours of type $A$ such that cach $\Delta_{i}$ intersects $\Gamma_{1}$ and the sum $\sum_{V}^{(2)}$ takes over all contours $\bar{\Lambda}$ of type $A$ which surrounds the contour $\Gamma_{1}$.

Let $N_{k}$ be the set of all $k$-ordered pairs of $A$-type contours $\left(\bar{\Gamma}_{1}, \ldots, \bar{\Gamma}_{k}\right)$ mutually disjoint in $Z^{2}$. Put

$$
\mathscr{H}=\left\{\Phi=\left(\phi_{k}\right)_{k \geqq 1} ;\|\Phi\|<\infty\right\}
$$

where $\phi_{k}: N_{h} \rightarrow R$ and

$$
\|\Phi\|=\sup _{k \geqq 1}\left[\sup _{\left(\bar{\Gamma}_{1}, \ldots, \bar{\Gamma}_{k}\right) \in N_{h}}\left|\phi_{k}\left(\bar{\Gamma}_{1}, \ldots, \bar{\Gamma}_{h}\right)\right| \prod_{i=1}^{h} \exp \left(\beta E\left(\bar{\Gamma}_{i}\right)\right) 2^{-\left|\Gamma_{i}\right|}\right] .
$$

Then $\mathscr{H}$ becomes a Banach space. Let $A$ be the linear operator given by

$$
\left\{\begin{array}{l}
(A \Phi)_{s}\left(\bar{\Gamma}_{1}, \ldots, \bar{\Gamma}_{s}\right)=\exp \left(-\beta E\left(\bar{\Gamma}_{1}\right)\right)\left\{\phi_{s-1}\left(\bar{\Gamma}_{2}, \ldots, \bar{\Gamma}_{s}\right)\right. \\
\quad+\sum_{k=1}^{\infty} \frac{(-1)^{k}}{k !} \sum_{Z^{2}, k}^{(1)} \phi_{s+h-1}\left(\bar{\Gamma}_{2}, \ldots, \bar{\Gamma}_{s}, \bar{\Delta}_{1}, \ldots, \bar{\Delta}_{k}\right) \\
\left.\quad-\sum_{Z^{2}}^{(2)} \phi_{s}\left(\bar{\Gamma}_{2}, \ldots, \bar{\Gamma}_{s}, \bar{\Lambda}\right)\right\} \quad \text { if } s>1 \\
(A \Phi)_{1}\left(\bar{\Gamma}_{1}\right)=\exp \left(-\beta E\left(\bar{\Gamma}_{1}\right)\right)\left\{\sum_{k=1}^{\infty} \frac{(-1)^{k}}{k !} \sum_{Z^{2}, k}^{(1)} \phi_{k}\left(\bar{\Delta}_{1} \ldots, \Delta_{k}\right)\right. \\
\left.-\sum_{Z^{2}}^{(2)} \phi_{1}(\bar{\Lambda})\right\} \quad \text { if } s=1
\end{array}\right.
$$

where the $\operatorname{sum} \sum_{Z^{2}, k}^{(1)}$ takes over all $k$-ordered pairs $\left(\Delta_{1}, \ldots, \Delta_{k}\right)$ such that $\left(\bar{\Gamma}_{2}, \ldots, \bar{\Gamma}_{s}, \bar{\Delta}_{1}, \ldots, \bar{\Delta}_{k}\right) \in N_{k+s-1}$ and each $\Delta_{m}$ intersects $\Gamma_{1}$, and the sum $\sum_{Z^{2}}^{(2)}$ takes over all contours of type $A$ such that $\left(\bar{\Gamma}_{2}, \ldots, \bar{\Gamma}_{s}, \bar{\Lambda}\right) \in N_{s}$ and $\Lambda$ surrounds $\Gamma_{1}$.

Then the correlation equations in $Z^{2}$ is expressed in the following equation on $\mathscr{M}$,

$$
\rho=A \rho+\Lambda
$$

where $A$ is the element of $\mathscr{H}$ given by 


$$
\Lambda\left(\bar{\Gamma}_{1}, \ldots, \bar{\Gamma}_{s}\right)=\left\{\begin{array}{cl}
\exp \left(-\beta E\left(\bar{\Gamma}_{1}\right)\right) & \text { if } s=1 \\
0 & \text { otherwise. }
\end{array}\right.
$$

When the norm of $A$ is smaller than one, the equation (3.3) has the unique solution. We call its solution "correlation functions in $\mathbb{Z}^{2}$ ".

We state the various properties of correlation functions in the following several lemmas. The proof of lemmas will be given in Section 4.

First of all we give the estimate of $\tau_{V, A}\left(\bar{\Gamma}_{1}, \ldots, \bar{\Gamma}_{s}\right)$ from above.

\section{Lemma 3.1.}

$$
\tau_{V, A}\left(\bar{\Gamma}_{1}, \ldots, \bar{\Gamma}_{s}\right) \leqq \prod_{i=1}^{s} \exp \left(-\beta E\left(\bar{\Gamma}_{i}\right)\right)
$$

As for the correlation function of outer contours, we have the same estimate.

\section{Lemma 3.2.}

$$
\rho_{V, A}\left(\bar{\Gamma}_{1}, \ldots, \bar{\Gamma}_{s}\right) \leqq \prod_{i=1}^{s} \exp \left(-\beta E\left(\bar{\Gamma}_{i}\right)\right)
$$

Let $\chi_{V, A}$ be the linear operator on $\mathscr{M}$ given by

$$
\begin{aligned}
& \left(\chi_{V, A}\right)_{k}\left(\bar{\Gamma}_{1}, \ldots, \bar{\Gamma}_{k}\right) \\
& \quad=\left\{\begin{array}{cl}
\phi_{k}\left(\bar{\Gamma}_{1}, \ldots, \bar{\Gamma}_{k}\right) & \text { if }\left(\bar{\Gamma}_{1}, \ldots, \bar{\Gamma}_{k}\right) \text { is admissible with }(V, A) \\
0 & \text { otherwise, }
\end{array}\right.
\end{aligned}
$$

From Lemma 3.2. we have $\chi_{V, A} \rho_{V, A} \in \mathscr{M} . \quad$ Further the correlation equations in $V$ is expressed in the following formula

$$
\chi_{V, A} \rho_{V, A}=\chi_{V, A} A \chi_{V, A} \rho_{V, A}+\chi_{V, A} \Lambda .
$$

Concerning the estimate of $\|A\|$ we have the following lemma.

Lemma 3.3. For sufficiently large $\beta$, we have

$$
\|A\|<1 \text {. }
$$

From this Lemma we have the unique solution of (3.4), and denote it by $\rho_{A}(\cdot)$.

Next, we estimate the following differences between correlation functions. Let $V_{1} \subset V_{2}$ be two finite subsets of $\mathbb{Z}^{2}$. Put

$$
\begin{aligned}
& \eta_{V_{1}, V_{2}, A}=\chi_{V_{1}, A} \rho_{V_{2}, A}-\chi_{V_{1}, A} \rho_{V_{1}, A} \\
& \eta_{V, Z^{2}, A}=\chi_{V, A} \rho_{A}-\chi_{V, A} \rho_{V, A}
\end{aligned}
$$

Lemma 3.4. For sufficiently large $\beta$ the following estimate holds,

1) $\left|\eta_{V_{1}, V_{2}, A}\left(\bar{\Gamma}_{1}, \ldots, \bar{\Gamma}_{s}\right)\right|<c_{1} \prod_{i=1}^{s} a\left(\bar{\Gamma}_{i}\right) \exp \left[-(3 / 4) \beta \varepsilon d\left(\Gamma_{1}, \ldots, \Gamma_{s} ; \partial\left(V_{1}, V_{2}\right)\right)\right]$ 
2) $\left|\eta_{V, Z^{2}, A}\left(\bar{\Gamma}_{1}, \ldots, \bar{\Gamma}_{s}\right)\right|<c_{1} \prod_{i=1}^{s} a\left(\bar{\Gamma}_{i}\right) \exp \left[-(3 / 4) \beta \varepsilon d\left(\Gamma_{1}, \ldots, \Gamma_{s} ; \partial V\right)\right]$ where $c_{1}$ is some absolute constant, $\varepsilon=\operatorname{Min}_{a, b \in S} \varepsilon(a, b), a(\bar{\Gamma})=2^{|\Gamma|} e^{-\beta E(\bar{\Gamma})}$, $\partial\left(V_{1}, V_{2}\right)=\partial V_{1} \backslash \partial V_{2}$, and $d\left(\Gamma_{1}, \ldots, \Gamma_{s} ; \partial\left(V_{1}, V_{2}\right)\right)$ is the distance between $\left\{\Gamma_{1}\right.$, $\left.\ldots, \Gamma_{s}\right\}$ and $\partial\left(V_{1}, V_{2}\right)$.

By using this lemma, we have the clustering property of correlation functions of outer contours.

Lemma 3.5. For sufficiently large $\beta$, the following estimate holds,

$$
\begin{aligned}
\mid \rho_{V, A}\left(\bar{\Gamma}_{1}, \bar{\Gamma}_{2}\right)-\rho_{V, A}( & \left.\bar{\Gamma}_{1}\right) \rho_{V, A}\left(\bar{\Gamma}_{2}\right) \mid \\
& <c_{2} a\left(\bar{\Gamma}_{1}\right) a\left(\bar{\Gamma}_{2}\right) \exp \left(-(3 / 4) \beta \varepsilon d\left(\Gamma_{1}, \Gamma_{2}\right)\right),
\end{aligned}
$$

where $c_{2}$ is the absolute constant.

As for the correlation function $\tau_{V . A}\left(\check{\Gamma}_{1}, \ldots, \bar{\Gamma}_{s}\right)$, we have the similar properties. Let $V_{1} \subset V_{2}$ be two finite subsets of $\mathbb{Z}^{2}$.

Lemma 3.6. For sufficiently large $\beta$, the following estimate holds,

$$
\left|\tau_{V_{1}, A}(\bar{\Gamma})-\tau_{V_{2}, A}(\bar{\Gamma})\right|<c_{3} a(\bar{\Gamma}) \exp \left[-(3 / 4) \beta \varepsilon d\left(\Gamma, \partial\left(V_{1}, V_{2}\right)\right)\right]
$$

whenever $\bar{\Gamma}$ is admissible with $\left(V_{1}, A\right)$, where $c_{3}$ is the absolute constant.

Similarly to Lemma 3.5 , we have the following lemma.

Lemma 3.7. For sufficiently large $\beta$, we have

$$
\left|\tau_{V, A}\left(\bar{\Gamma}_{1}, \bar{\Gamma}_{2}\right)-\tau_{V, A}\left(\bar{\Gamma}_{1}\right) \tau_{V, A}\left(\bar{\Gamma}_{2}\right)\right|<c_{4} a\left(\bar{\Gamma}_{1}\right) a\left(\bar{\Gamma}_{2}\right) \exp \left[-(3 / 4) \beta \varepsilon d\left(\Gamma_{1}, \Gamma_{2}\right)\right]
$$

whenever $\left(\bar{\Gamma}_{1}, \bar{\Gamma}_{2}\right)$ is admissible with $(V, A)$ and $v\left(\Gamma_{1}\right) \cap v\left(\Gamma_{2}\right)=\varnothing$, where $c_{4}$ is the absolute constant.

\section{§4. Proof of Lemmas}

In this section we give the proof of lemmas stated in Section 3.

First we prove Lemma 3.1 in the case of $s=1$. We define the mapping $g_{\bar{\Gamma}_{1}}$ from $\mathscr{B}\left(\bar{\Gamma}_{1}\right)$ to $\Omega_{V, A}$ as follows. When $\zeta \in \mathscr{B}\left(\bar{\Gamma}_{1}\right)$ is denoted by

$$
\dot{\zeta}=\left(\bar{\Gamma}_{1}, \bar{\Delta}_{1}, \ldots, \bar{\Delta}_{m}, \bar{\Lambda}_{1}, \ldots, \bar{\Lambda}_{s}\right) \text {, }
$$

$g_{\bar{\Gamma}_{1}}(\xi)$ is given by

$$
g_{\bar{\Gamma}_{1}}(\xi)=\left(f_{\bar{\Gamma}_{1}}\left(\bar{\Delta}_{1}\right), \ldots, f_{\bar{\Gamma}_{1}}\left(\bar{\Delta}_{m}\right), \bar{\Lambda}_{1}, \ldots, \bar{\Lambda}_{s}\right)
$$

where $\left\{\bar{\Delta}_{1}, \ldots, \bar{\Delta}_{m}\right\}$ is the totality of contours included in $\theta\left(\Gamma_{1}\right)$ and $\left\{\bar{\Lambda}_{1}, \ldots, \bar{\Lambda}_{s}\right\}$ 
is the totality of contours included in $V \backslash w\left(\Gamma_{1}\right)$. It is easily seen that $g_{\bar{\Gamma}_{1}}$ is one-to-one as the mapping from $\mathscr{B}\left(\bar{\Gamma}_{1}\right)$ to $\Omega_{V, A}$ and

$$
U_{V}\left(g_{\bar{\Gamma}_{1}}(\xi)\right)=U_{V}(\xi)-E\left(\bar{\Gamma}_{1}\right)
$$

By using the facts above, we have

$$
\begin{aligned}
\sum_{\xi \in:\left(\bar{\Gamma}_{1}\right)} \exp \left\{-\beta U_{V}(\xi)\right\} & =\exp \left\{-\beta E\left(\bar{\Gamma}_{1}\right)\right\} \sum_{\xi \in \mathscr{B}\left(\bar{\Gamma}_{1}\right)} \exp \left\{-\beta U_{V}\left(g_{\bar{\Gamma}_{1}}(\xi)\right)\right\} \\
& <\exp \left\{-\beta E\left(\bar{\Gamma}_{1}\right)\right\} \sum_{\zeta \in \Omega_{V}, A} \exp \left\{-\beta U_{V}(\zeta)\right\}
\end{aligned}
$$

Hence,

$$
\begin{aligned}
\tau_{V, A}\left(\bar{\Gamma}_{1}\right) & =\sum_{\xi \in \mathscr{g}\left(\bar{\Gamma}_{1}\right)} \exp \left(-\beta U_{V}(\zeta)\right) / \sum_{\xi \in \Omega_{V, A}} \exp \left(-\beta U_{V}(\check{\zeta})\right) \\
& <\exp \left(-\beta E\left(\bar{\Gamma}_{1}\right)\right) .
\end{aligned}
$$

In general case, we can prove it by composing the mappings $g_{\bar{\Gamma}_{1}}, \ldots, g_{\bar{\Gamma}_{s}}$.

In the same way we can prove Lemma 3.2.

Before proving Lemma 3.3. we prepare the following lemma which will be used many times in the sequel.

Lemma 4.1. For sufficiently large $\beta$, the following estimates hold,

1) $\quad \sum_{\bar{\Gamma}: \Gamma \ni 0} a(\bar{\Gamma}) \leqq h(\beta) \quad(h(\beta) / \exp (-3 \beta \varepsilon) \downarrow 0$ as $\beta \rightarrow \infty)$

2) $\sum_{\bar{\Gamma}: \Gamma \ni 0,|\Gamma| \geqq l} a(\bar{\Gamma}) \leqq d_{1} \exp \left(-\frac{3}{4} \beta \varepsilon l\right)$

3) $\quad \sum_{\bar{\Gamma} \in \bar{\gamma}: \bar{\Gamma} \subset \theta} a(\bar{\Gamma}) \leqq|\theta| 2|\gamma| \exp (-\beta E(\bar{\gamma}))$

4) $\quad \sum_{\bar{\Gamma}: \bar{\Gamma} \subset \theta} a(\bar{\Gamma}) \leqq$ const. $|\theta| \exp (-3 \beta \varepsilon)$

where $d_{1}$ is the absolute constant and $\varepsilon=\operatorname{Min} \varepsilon(a, b)$.

Taking into account the fact that

$$
E(\bar{\Gamma}) \geqq(|\Gamma|-1 / 4|\Gamma|) \varepsilon
$$

we can prove this lemma by the standard argument.

Proof of Lemma 3.3. When $\|\Phi\|<1$, we have

$$
\left|\phi_{k}\left(\bar{\Gamma}_{1}, \ldots, \bar{\Gamma}_{k}\right)\right|<\prod_{i=1}^{k} a\left(\bar{\Gamma}_{i}\right)
$$

for all $k$ and any $\left(\bar{\Gamma}_{1}, \ldots, \bar{\Gamma}_{k}\right) \in N_{k}$. From Lemma 4.1 and (4.1), we have 


$$
\begin{aligned}
& (A \Phi)_{k}\left(\bar{\Gamma}_{1}, \ldots, \bar{\Gamma}_{k}\right) \prod_{i=1}^{k} a\left(\bar{\Gamma}_{i}\right)^{-1} \\
& \quad \leqq \frac{1}{2^{\left|\Gamma_{1}\right|}}\left\{1+\sum_{m=1}^{\left|\Gamma_{1}\right|}\left(\begin{array}{c}
\left|\Gamma_{1}\right| \\
m
\end{array}\right)\left(\sum_{\bar{\Gamma}: \Gamma_{\ni 0}} a(\bar{\Gamma})\right)^{m}+\sum_{p=1}^{\infty} \sum_{\bar{\Gamma}: \Gamma \ni 0,|\Gamma| \geqq 2 p} a(\bar{\Gamma})\right\} \\
& \leqq \frac{1}{2^{\left|\Gamma_{1}\right|}}\left\{(1+h(\beta))^{\left|\Gamma_{1}\right|}+d_{1} \sum_{p=1}^{\infty} \exp \left(-\frac{3}{2} \beta \varepsilon p\right)\right\} \\
& \quad \leqq \sup _{k \geqq 4}\left\{\left(\frac{1+h(\beta)}{2}\right)^{k}+\frac{2 d_{1}}{2^{k}} \exp \left(-\frac{3}{2}-\beta \varepsilon p\right)\right\} \\
& \quad<1
\end{aligned}
$$

for sufficiently large $\beta$. Therefore Lemma 3.3 was proved.

Before proving Lemmas 3.4-3.6, we prepare the following lemma. For a given subset $S$ of $Z^{2}$, we define the Banach space $\mathscr{M}(S)$ by

where

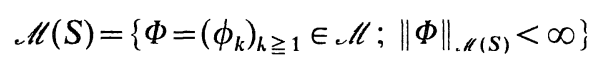

$$
\|\Phi\|_{\mathscr{U}(S)}=\sup _{k \geqq 4}\left[\sup _{\left(\bar{\Gamma}_{1}, \ldots, \bar{\Gamma}_{k}\right)}\left|\phi_{k}\left(\bar{\Gamma}_{1}, \ldots, \bar{\Gamma}_{k}\right)\right| \prod_{i=1}^{k} a\left(\bar{\Gamma}_{i}\right)^{-1} e^{(3 / 4) \beta \varepsilon d\left(\Gamma_{1}, \ldots, \Gamma_{k}: S\right)}\right]
$$

Lemma 4.2. 1) If $\Phi \in \mathscr{M}(S)$, then $A \Phi \in \mathscr{M}(S)$.

2) $\|A\|_{\mathscr{v}(S)}<1$ for sufficiently large $\beta$.

We can prove this lemma by the similar way to the proof of Lemma 3.3 of [2].

Now we prove Lemma 3.4. From the following two correlation equations

$$
\begin{aligned}
& \chi_{V_{1}, A} \rho_{V_{1}, A}=\chi_{V_{1}, A} A \chi_{V_{1}, A} \rho_{V_{1}, A}+\chi_{V_{1}, A} \Lambda \\
& \chi_{V_{2}, A} \rho_{V_{2}, A}=\chi_{V_{2}, A} A \chi_{V_{2}, A} \rho_{V_{2}, A}+\chi_{V_{2}, A} \Lambda
\end{aligned}
$$

we have

$$
\eta_{V_{1}, V_{2}, A}=\chi_{V_{1}, A} A \eta_{V_{1}, V_{2}, A}+\zeta_{V_{1}, V_{2}, A}
$$

where

$$
\zeta_{V_{1}, V_{2}, A}=\chi_{V_{1}, A} A\left(\chi_{V_{2}, A} \rho_{V_{2}, A}-\chi_{V_{1}, A} \rho_{V_{2}, A}\right) .
$$

In the similar way to the proof of Lemma 3.4 of [2], we have the following estimate of $\zeta_{V_{1}, V_{2}, A}\left(\bar{\Gamma}_{1}, \ldots, \bar{\Gamma}_{s}\right)$,

$$
\begin{aligned}
& \left|\zeta_{V_{1}, V_{2}, A}\left(\bar{\Gamma}_{1}, \ldots, \bar{\Gamma}_{s}\right)\right| \\
& \quad<d_{2} \prod_{i=1}^{s} a\left(\bar{\Gamma}_{i}\right) \exp \left(-\frac{3}{2} \beta \varepsilon d\left(\Gamma_{1}, \partial\left(V_{1}, V_{2}\right)\right)\right)
\end{aligned}
$$

where $d_{2}$ is the absolute constant. Hence, $\zeta_{V_{1}, V_{2}, A} \in \mathscr{M}\left(\partial\left(V_{1}, V_{2}\right)\right)$ and $\left\|\zeta_{V_{1}, V_{2}, A}\right\|_{\mathscr{M}(S)}<d_{2}$. 
From Lemma 4.2 and (4.4), we have

$$
\begin{aligned}
& \mid \eta_{V_{1}, V_{2}, A}\left(\bar{\Gamma}_{1}, \ldots, \bar{\Gamma}_{s}\right)<\frac{d_{2}}{1-\|A\|_{\mathscr{M}\left(\partial\left(V_{1}, V_{2}\right)\right)}} \\
& \quad \times \prod_{i=1}^{s} a\left(\bar{\Gamma}_{i}\right) \cdot \exp \left[-\frac{3}{4} \beta \varepsilon d\left(\Gamma_{1}, \ldots, \Gamma_{s} ; \partial\left(V_{1}, V_{2}\right)\right)\right] .
\end{aligned}
$$

Therefore the first assertion of Lemma 3.4 was proved. In the same way we can prove the second assertion.

Proof of Lemma 3.5. From the definition of correlation functions of outer contours, we have

$$
\begin{aligned}
& \left|\rho_{V, A}\left(\bar{\Gamma}_{1}, \bar{\Gamma}_{2}\right)-\rho_{V, A}\left(\bar{\Gamma}_{1}\right) \rho_{V, A}\left(\bar{\Gamma}_{2}\right)\right| \\
& \quad=\rho_{V, A}\left(\bar{\Gamma}_{1}\right)\left|\rho_{V \backslash W\left(\Gamma_{1}\right), A}\left(\bar{\Gamma}_{2}\right)-\rho_{V, A}\left(\bar{\Gamma}_{2}\right)\right| .
\end{aligned}
$$

From Lemma 3.4, we have

$$
\begin{aligned}
& \left|\rho_{V, A}\left(\bar{\Gamma}_{1}, \bar{\Gamma}_{2}\right)-\rho_{V, A}\left(\bar{\Gamma}_{1}\right) \rho_{V, A}\left(\bar{\Gamma}_{2}\right)\right| \\
& \quad<c_{1} a\left(\bar{\Gamma}_{1}\right) a\left(\bar{\Gamma}_{2}\right) \exp \left[-\frac{3}{4} \beta \varepsilon d\left(\bar{\Gamma}_{1}, \Gamma_{2}\right)\right] .
\end{aligned}
$$

The proof of Lemma 3.6 is divided into following two cases.

Case $I . \quad o(\bar{\Gamma}) \neq A$.

In this case $\bar{\Gamma}$ cannot be the outer contour. As $\Gamma$ is surrounded by some outer contour $\bar{\Gamma}_{1}$, we have

$$
\tau_{V, A}(\bar{\Gamma})=\sum_{\bar{\Gamma}_{1}: o\left(\bar{\Gamma}_{1}\right)=A, \theta\left(\Gamma_{1}\right) \supset \theta(\Gamma)} \rho_{V, A}\left(\bar{\Gamma}_{1}\right) \tau_{\theta\left(\Gamma_{1}\right), i\left(\bar{\Gamma}_{1}\right)}(\bar{\Gamma}) .
$$

By using this formula, we have

$$
\begin{aligned}
& \left|\tau_{V_{1}, A}(\bar{\Gamma})-\tau_{V_{2}, A}(\bar{\Gamma})\right| \\
& \quad \leqq \\
& \quad \sum_{\bar{\Gamma}_{1}: o\left(\bar{\Gamma}_{1}\right)=A, V_{1} \supset \theta\left(\Gamma_{1}\right) \supset \theta(\Gamma)}\left|\rho_{V_{1}, A}\left(\bar{\Gamma}_{1}\right)-\rho_{V_{2}, A}\left(\bar{\Gamma}_{1}\right)\right| \tau_{\theta\left(\Gamma_{1}\right), i\left(\bar{\Gamma}_{1}\right)}(\bar{\Gamma}) \\
& \quad+\sum_{\bar{\Gamma}_{1}: o\left(\bar{\Gamma}_{1}\right)=A, \theta\left(\Gamma_{1}\right) \supset \theta(\Gamma), \theta\left(\Gamma_{1}\right) \cap \partial\left(V_{1}, V_{2}\right) \neq \emptyset} \rho_{V_{2}, A}\left(\bar{\Gamma}_{1}\right) \tau_{\theta\left(\Gamma_{1}\right), i\left(\bar{\Gamma}_{1}\right)}(\bar{\Gamma}) .
\end{aligned}
$$

From Lemma 3.4, the first term is estimated as follows.

$$
\begin{aligned}
I_{1} & \leqq c_{1} a(\bar{\Gamma}) \sum_{\bar{\Gamma}_{1}: o\left(\bar{\Gamma}_{1}\right)=A, V_{1} \supset \theta\left(\Gamma_{1}\right) \supset \theta(\Gamma)} a\left(\bar{\Gamma}_{1}\right) \exp \left[-\frac{3}{4} \beta \varepsilon d\left(\Gamma_{1}, \partial\left(V_{1}, V_{2}\right)\right)\right] \\
& \leqq c_{1} a(\bar{\Gamma}) \sum_{p=1}^{d\left(\Gamma, \partial\left(V_{1}, V_{2}\right)\right)-1} e^{-(3 / 4) \beta \varepsilon p} \sum_{\bar{\Gamma}_{1}: \Gamma_{1} \ni 0,\left|\Gamma_{1}\right|>2\left(d\left(\Gamma, \partial\left(V_{1}, V_{2}\right)\right)-p\right)} a\left(\bar{\Gamma}_{1}\right) \\
& \geqq c_{1} d_{1} a(\bar{\Gamma}) \sum_{p=1}^{d\left(\Gamma, \partial\left(V_{1}, V_{2}\right)\right)-1} e^{-(3 / 4) \beta \varepsilon p} \exp \left[-\beta \varepsilon\left(d\left(\Gamma, \partial\left(V_{1}, V_{2}\right)\right)-p\right)\right] \\
& \leqq c_{1} d_{1} a(\bar{\Gamma}) \exp \left[-\frac{3}{4} \beta \varepsilon d\left(\Gamma, \partial\left(V_{1}, V_{2}\right)\right)\right] \sum_{p=1}^{\infty} e^{-(1 / 4) \beta \varepsilon p} .
\end{aligned}
$$


By using the same argument as in the proof of Lemma 3.4 in [2], we have

$$
\begin{aligned}
I_{2} & \leqq \sum_{k=1}^{\infty} \sum_{\bar{\Gamma}_{1}: \Gamma_{1} \ni 0,\left|\Gamma_{1}\right|>2\left(d\left(\Gamma, \hat{a}\left(V_{1}, V_{2}\right)\right)+k\right)} a\left(\bar{\Gamma}_{1}\right) a(\bar{\Gamma}) \\
& \leqq a(\bar{\Gamma}) \sum_{k=1}^{\infty} d_{1} \exp \left(-\frac{3}{2} \beta \varepsilon\left(d\left(\Gamma, \partial\left(V_{1}, V_{2}\right)\right)+k\right)\right) \\
& \leqq d_{1} a(\bar{\Gamma}) \exp \left[-\frac{3}{2} \beta \varepsilon d\left(\Gamma, \partial\left(V_{1}, V_{2}\right)\right)\right] \sum_{k=1}^{\infty} e^{-(3 / 2) \beta \varepsilon k}
\end{aligned}
$$

From these estimates the assertion of Lemma 3.6 is proved.

Case II. $o(\bar{\Gamma})=A$.

In this case $\tau_{V, A}(\bar{\Gamma})$ is expressed in the following formula.

$$
\tau_{V, A}(\bar{\Gamma})=\rho_{V, A}(\bar{\Gamma})+\sum_{\substack{\left.\bar{T}_{1}: \rho_{(\bar{\Gamma}}\right)=A \\ \theta\left(\Gamma_{1}\right)>\theta(\Gamma)}} \rho_{V, A}\left(\bar{\Gamma}_{1}\right) \tau_{\theta\left(\Gamma_{1}\right), i\left(\bar{\Gamma}_{1}\right)}(\bar{\Gamma})
$$

By using this formula and the same argument in Case I, we obtain the assertion of Lemma 3.6.

Finally we prove Lemma 3.7. The proof of this lemma is also divided into following two cases.

Case I. $o\left(\bar{\Gamma}_{1}\right)=A$ or $o\left(\bar{\Gamma}_{2}\right)=A$.

Case II. $o\left(\bar{\Gamma}_{1}\right) \neq A$ and $o\left(\bar{\Gamma}_{2}\right) \neq A$.

Case I. $o\left(\bar{\Gamma}_{1}\right)=A$ or $o\left(\vec{\Gamma}_{2}\right)=A$.

When $o\left(\bar{\Gamma}_{1}\right)=A$, we have

$$
\begin{aligned}
& \left|\tau_{V, A}\left(\bar{\Gamma}_{1}, \bar{\Gamma}_{2}\right)-\tau_{V, A}\left(\bar{\Gamma}_{1}\right) \tau_{V, A}\left(\bar{\Gamma}_{2}\right)\right| \\
& \quad=\tau_{V, A}\left(\bar{\Gamma}_{1}\right)\left|\tau_{V \mid W\left(\Gamma_{1}\right), A}\left(\bar{\Gamma}_{2}\right)-\tau_{V, A}\left(\bar{\Gamma}_{2}\right)\right|
\end{aligned}
$$

From Lemma 3.6, we have

$$
\begin{aligned}
& \left|\tau_{V, A}\left(\bar{\Gamma}_{1}, \bar{\Gamma}_{2}\right)-\tau_{V, A}\left(\bar{\Gamma}_{1}\right) \tau_{V, A}\left(\bar{\Gamma}_{2}\right)\right| \\
& \quad<c_{1} a\left(\bar{\Gamma}_{1}\right) a\left(\bar{\Gamma}_{2}\right) \exp \left[-\frac{3}{4} \beta \varepsilon d\left(\Gamma_{1}, \Gamma_{2}\right)\right]
\end{aligned}
$$

Case II. $o\left(\bar{\Gamma}_{1}\right) \neq A$ and $o\left(\bar{\Gamma}_{2}\right) \neq A$.

As both $\bar{\Gamma}_{1}$ and $\bar{\Gamma}_{2}$ are not outer contours, there exist outer contours which surround $\Gamma_{1}$ and $\Gamma_{2}$. We denote the set of all contours of type $A$ which surrounds $\Gamma_{1}$ and does not surround $\Gamma_{2}$ by $C_{1}\left(\bar{\Gamma}_{1}, \bar{\Gamma}_{2}\right)$. For each $\bar{\Gamma} \in C_{1}\left(\bar{\Gamma}_{1}, \bar{\Gamma}_{2}\right)$, we denote the set of all configurations in $\mathscr{B}\left(\bar{\Gamma}_{1}, \bar{\Gamma}_{2}\right)$ which contains $\bar{\Gamma}$ as an outer contour by $\mathscr{B}_{1}\left(\bar{\Gamma}_{1}, \bar{\Gamma}_{2} ; \vec{\Gamma}\right)$. We also denote the set of all contours of type $A$ which surround $\Gamma_{1}$ and $\Gamma_{2}$ together by $C_{2}\left(\bar{\Gamma}_{1}, \bar{\Gamma}_{2}\right)$. For each $\bar{\Gamma} \in C_{2}\left(\bar{\Gamma}_{1}, \bar{\Gamma}_{2}\right)$, we denote the set of all configurations in $\mathscr{B}\left(\bar{\Gamma}_{1}, \bar{\Gamma}_{2}\right)$ which contains $\bar{\Gamma}$ as an 
outer contour by $\mathscr{B}_{2}\left(\bar{\Gamma}_{,} \bar{\Gamma}_{2} ; \bar{\Gamma}\right)$. Then we have

$$
\mathscr{B}\left(\bar{\Gamma}_{1}, \bar{\Gamma}_{2}\right)=\sum_{\bar{\Gamma} \in C_{1}\left(\bar{\Gamma}_{1}, \bar{\Gamma}_{2}\right)} \mathscr{B}_{1}\left(\bar{\Gamma}_{1}, \bar{\Gamma}_{2} ; \bar{\Gamma}\right)+\sum_{\bar{\Gamma} \in C_{2}\left(\bar{\Gamma}_{1}, \bar{\Gamma}_{2}\right)} \mathscr{B}_{2}\left(\bar{\Gamma}_{1}, \bar{\Gamma}_{2} ; \bar{\Gamma}\right) .
$$

Further we denote the set of all contours of type $A$ which surround $\Gamma_{1}$ and intersect with $\Gamma_{2}$ by $C_{3}\left(\bar{\Gamma}_{1}, \bar{\Gamma}_{2}\right)$. For each $\bar{\Gamma} \in C_{1}\left(\bar{\Gamma}_{1}, \bar{\Gamma}_{2}\right) \cup C_{2}\left(\bar{\Gamma}_{1}, \bar{\Gamma}_{2}\right)$ $\cup C_{3}\left(\bar{\Gamma}_{1}, \bar{\Gamma}_{2}\right)$, we denote the set of all configurations in $\mathscr{B}\left(\bar{\Gamma}_{1}\right)$ which contains $\bar{\Gamma}$ as an outer contour by $\mathscr{D}\left(\bar{\Gamma}_{1} ; \bar{\Gamma}\right)$. Then we have

$$
\mathscr{B}\left(\bar{\Gamma}_{1}\right)=\sum_{\bar{\Gamma} \in C_{1}\left(\bar{\Gamma}_{1}, \bar{\Gamma}_{2}\right)} \mathscr{D}\left(\bar{\Gamma}_{1} ; \bar{\Gamma}\right)+\sum_{\bar{\Gamma} \in C_{2}\left(\bar{\Gamma}_{1}, \bar{\Gamma}_{2}\right)} \mathscr{D}\left(\bar{\Gamma}_{1} ; \bar{\Gamma}\right)+\sum_{\bar{\Gamma}_{\in} \in C_{3}\left(\bar{\Gamma}_{1}, \bar{\Gamma}_{2}\right)} \mathscr{D}\left(\bar{\Gamma}_{1} ; \bar{\Gamma}\right) .
$$

Hence, we have the following estimate,

$$
\begin{aligned}
& \left|\tau_{V, A}\left(\bar{\Gamma}_{1}, \bar{\Gamma}_{2}\right)-\tau_{V, A}\left(\bar{\Gamma}_{1}\right) \tau_{V, A}\left(\bar{\Gamma}_{2}\right)\right| \\
& \quad \leqq \\
& \quad \sum_{\bar{\Gamma} \in C_{1}\left(\bar{\Gamma}_{1}, \bar{\Gamma}_{2}\right)}\left|P_{V, A}\left(\mathscr{B}_{1}\left(\bar{\Gamma}_{1}, \bar{\Gamma}_{2} ; \bar{\Gamma}\right)\right)-P_{V, A}\left(\mathscr{D}\left(\bar{\Gamma}_{1} ; \bar{\Gamma}\right)\right) P_{V, A}\left(\mathscr{B}\left(\bar{\Gamma}_{2}\right)\right)\right| \\
& \quad+\sum_{\bar{\Gamma}_{\in} C_{2}\left(\bar{\Gamma}_{1}, \bar{\Gamma}_{2}\right)}\left|P_{V, A}\left(\mathscr{B}_{2}\left(\bar{\Gamma}_{1}, \bar{\Gamma}_{2} ; \bar{\Gamma}\right)\right)-P_{V, A}\left(\mathscr{D}\left(\bar{\Gamma}_{1} ; \bar{\Gamma}\right)\right) P_{V, A}\left(\mathscr{B}\left(\bar{\Gamma}_{2}\right)\right)\right| \\
& \quad+\sum_{\bar{\Gamma} \in C_{3}\left(\bar{\Gamma}_{1}, \bar{\Gamma}_{2}\right)} P_{V, A}\left(\mathscr{D}\left(\bar{\Gamma}_{1}, \bar{\Gamma}\right)\right) P_{V, A}\left(\mathscr{B}\left(\bar{\Gamma}_{2}\right)\right) .
\end{aligned}
$$

We denote the first, second and third terms in the right-hand side by $I_{1}, I_{2}$, and $I_{3}$. We estimate them seperately in the following.

First of all we estimate the first term $I_{1}$. From Lemma 3.6, we have

$$
\begin{aligned}
I_{1} & =\sum_{\bar{\Gamma} \in C_{1}\left(\bar{\Gamma}_{1}, \bar{\Gamma}_{2}\right)} P_{V, A}\left(\mathscr{D}\left(\bar{\Gamma}_{1} ; \bar{\Gamma}\right)\right)\left|P_{V, A}\left(\mathscr{B}_{1}\left(\bar{\Gamma}_{1}, \bar{\Gamma}_{2} ; \bar{\Gamma}\right) \mid \mathscr{D}\left(\bar{\Gamma}_{1} ; \bar{\Gamma}\right)\right)-P_{V, A}\left(\mathscr{B}\left(\bar{\Gamma}_{2}\right)\right)\right| \\
& \leqq \sum_{\bar{\Gamma}_{\in} C_{1}\left(\bar{\Gamma}_{1}, \bar{\Gamma}_{2}\right)} \exp \left(-\beta\left(E\left(\bar{\Gamma}_{1}\right)+E(\bar{\Gamma})\right)\right)\left|\tau_{V \mid W(\Gamma), A}\left(\bar{\Gamma}_{2}\right)-\tau_{V, A}\left(\bar{\Gamma}_{2}\right)\right| \\
& \leqq c_{3} a\left(\bar{\Gamma}_{2}\right) e^{-\beta E\left(\bar{\Gamma}_{1}\right)} \sum_{\bar{\Gamma}_{\in} C_{1}\left(\bar{\Gamma}_{1}, \bar{\Gamma}_{2}\right)} e^{-\beta E(\bar{\Gamma})} \exp \left(-\frac{3}{4} \beta \varepsilon d\left(\Gamma, \Gamma_{2}\right)\right) \\
& \leqq c_{3}\left|\Gamma_{1}\right| e^{-\beta E\left(\bar{\Gamma}_{1}\right)} a\left(\bar{\Gamma}_{2}\right) \sum_{p=1}^{d\left(\Gamma_{1}, \Gamma_{2}\right)-1} e^{-(3 / 4) \beta \varepsilon p} d \sum_{m=1}^{d\left(\Gamma_{1}, \Gamma_{2}\right)-p}(1+2 m) \cdot \\
& \leqq c_{3} d_{1} a\left(\bar{\Gamma}_{1}\right) a\left(\bar{\Gamma}_{2}\right) \frac{\left|\Gamma_{1}\right|}{2^{\left|I_{1}\right|}} \sum_{p=1}^{d\left(\Gamma_{1}, \Gamma_{2}\right)-1} e^{-(3 / 4) \beta \varepsilon p} \sum_{m=1}^{d\left(\Gamma_{1}, \Gamma_{2}\right)-p}(1+2 m) \cdot \\
& \leqq c_{3} d_{1} \cdot \sup _{k \geqq 4} \frac{k}{2^{k}} \cdot a\left(\bar{\Gamma}_{1}\right) a\left(\bar{\Gamma}_{2}\right) \cdot \exp \left(-\frac{3}{4} \beta \varepsilon d\left(\Gamma_{1}, \Gamma_{2}\right)\right) \sum_{p=1}^{\infty}\left(p^{2}+2 p\right) e^{-(3 / 4) \beta \varepsilon p}
\end{aligned}
$$

Here we have used the fact that the number of points satisfying $d\left(\Gamma_{1}, t\right)<$ $d\left(\Gamma_{1}, \Gamma_{2}\right)-p$ is at most

$$
\sum_{m=1}^{d\left(\Gamma_{1}, \Gamma_{2}\right)-p}\left(\left|\Gamma_{1}\right|+8 m\right) .
$$


Next we estimate the second term. As $|\Gamma|>2 d\left(\Gamma_{1}, \Gamma_{2}\right)$ for each $\bar{\Gamma} \in C_{2}\left(\bar{\Gamma}_{1}\right.$, $\bar{\Gamma}_{2}$ ), we have

$$
I_{2}<2 d_{1} \sum_{k=1}^{\infty} \exp (-\beta \varepsilon k) a\left(\bar{\Gamma}_{1}\right) a\left(\bar{\Gamma}_{2}\right) \exp \left(-\frac{3}{2} \beta \varepsilon d\left(\Gamma_{1}, \Gamma_{2}\right)\right)
$$

As for $I_{3}$, the similar estimate to $I_{2}$ is obtained. Hence, we have

$$
\begin{aligned}
& \left|\tau_{V, A}\left(\bar{\Gamma}_{1}, \bar{\Gamma}_{2}\right)-\tau_{V, A}\left(\bar{\Gamma}_{1}\right) \tau_{V, A}\left(\bar{\Gamma}_{2}\right)\right| \\
& \quad<c_{4} a\left(\bar{\Gamma}_{1}\right) a\left(\bar{\Gamma}_{2}\right) \exp \left[-\frac{3}{4} \beta \varepsilon d\left(\Gamma_{1}, \Gamma_{2}\right)\right]
\end{aligned}
$$

whenever $v\left(\Gamma_{1}\right) \cap v\left(\Gamma_{2}\right)=\varnothing$, where $c_{4}$ is the absolute constant.

\section{§5. The Expectation Value and the Variance of the Number of Particles}

In this section we estimate the expectation value and the variance of the number of particles by using the correlation functions.

We prepare some notations which will be used in this section. We say $\bar{\Gamma}_{1}$ is congruent to $\bar{\Gamma}_{2}$ if $o\left(\bar{\Gamma}_{1}\right)=o\left(\bar{\Gamma}_{2}\right), i\left(\bar{\Gamma}_{1}\right)=i\left(\bar{\Gamma}_{2}\right)$, and $\Gamma_{1}$ is superimposed on $\Gamma_{2}$ by translation. A congruent class is denoted by $\bar{\gamma}$, and the set of such congruence classes is denoted by $\Gamma$.

If $\bar{\Gamma}_{1}$ is congruent to $\bar{\Gamma}_{2}$, then

$$
\rho_{A}\left(\bar{\Gamma}_{1}\right)=\rho_{A}\left(\bar{\Gamma}_{2}\right) .
$$

From the standard argument, the expectation value of $N_{a}(\xi: V)$ is given by

$$
\left\langle N_{a}\right\rangle_{V, A}=\left\{\begin{array}{cc}
|V|-\sum_{\bar{\gamma}: o(\bar{\gamma})=A}\left(|V(\gamma)|-\left\langle n_{a}(\bar{\gamma})\right\rangle\right) \sum_{\substack{\bar{\Gamma} \in \bar{\gamma} \\
\Gamma \in V, A}} \rho_{V, A}(\bar{\Gamma}) \quad(a=A) \\
\sum_{\bar{\gamma}: o(\bar{\gamma})=A}\left\langle n_{a}(\bar{\gamma})\right\rangle \sum_{\substack{\bar{\Gamma} \in \bar{\gamma} \\
\Gamma \supset V}} \rho_{V, A}(\bar{\Gamma}) & (a \neq A)
\end{array}\right.
$$

where $\left\langle n_{a}(\bar{\gamma})\right\rangle$ is the expectation value of $N_{a}(\xi: v(\gamma))$ in the ensemble $\Omega_{v(\gamma), i(\bar{\gamma})}$. Put

$$
\rho_{1}^{*}=\sum_{\bar{\gamma}: o(\bar{\gamma})=a}\left(|V(\gamma)|-\left\langle n_{a}(\bar{\gamma})\right\rangle\right) \rho_{a}(\bar{\gamma})
$$

and $\rho_{1}^{* *}=1-\rho_{1}^{*}$. From the symmetry of interactions $\rho_{1}^{*}=\rho_{1}^{*}(\beta)$ is independent of $a$, and $\rho_{1}^{*}(\beta) / \exp (-3 \beta \varepsilon) \downarrow 0$ exponentially as $\beta \rightarrow \infty$, where $\varepsilon=\operatorname{Min}(\varepsilon(a, b))$.

From Lemma 3.7 and (5.1), we have

$$
\begin{aligned}
& \left|\left\langle N_{A}\right\rangle_{V, A}-\rho_{1}^{* *}\right| V|| \\
& \quad<\sum_{\bar{\gamma}: o(\bar{\gamma})=A}\left(|V(\gamma)|-\left\langle n_{A}(\bar{\gamma})\right\rangle\right)\left|\sum_{\substack{\bar{\Gamma} \in \bar{\gamma} \\
\Gamma \subset V}} \rho_{V \cdot A}(\bar{\Gamma})-\rho_{A}(\bar{\gamma})\right| V||
\end{aligned}
$$




$$
\begin{aligned}
& <\sum_{\bar{\gamma}: o(\bar{\gamma})=A}|\gamma|^{2}\left\{\sum_{\substack{\bar{T} \in \bar{\gamma} \\
x(\Gamma) \in V}}\left|\rho_{V, A}(\bar{\Gamma})-\rho_{A}(\bar{\gamma})\right|+\sum_{\substack{\bar{\Gamma} \in \bar{\gamma} \\
x(\Gamma) \in V, \Gamma \Phi V}} \exp (-\beta E(\bar{\Gamma}))\right\} \\
& <\sum_{\bar{\gamma}: o(\bar{\gamma})=A}\left\{\sum_{k=1}^{\infty}(|\partial V|+8 k) 6|\gamma| \cdot e^{-(3 / 4) \beta \varepsilon k}+|\partial V| \cdot|\gamma|\right\} e^{-\beta E(\bar{\gamma})}
\end{aligned}
$$

where $x(\Gamma)$ is the left most lowerst point in $\Gamma$. Hence, we have

$$
\left|\left\langle N_{A}\right\rangle_{V, A}-\rho_{1}^{* *}\right| V||<F_{1}(\beta)|\partial V|
$$

where

$$
F_{1}(\beta)=2 \sum_{\bar{\gamma}: o(\bar{\gamma})=A}|\gamma|^{3} 6^{\gamma} e^{-\beta E(\bar{\gamma})}, \quad F_{1}(\beta) / \exp (-3 \beta \varepsilon) \downarrow 0 \quad \text { as } \quad \beta \longrightarrow \infty .
$$

Put

$$
\rho_{2}^{*}=\sum_{\bar{\gamma}: o(\bar{\gamma})=a}\left\langle n_{b}(\bar{\gamma})\right\rangle \rho_{a}(\bar{\gamma}) \quad(a \neq b) .
$$

Then $\rho_{2}^{*}$ is also independent of $a$ and $b$. From Lemma 3.7 and (5.1) we have

$$
\begin{aligned}
& \quad\left\langle N_{b}\right\rangle_{V, A}-\rho_{2}^{*}|V| \mid \\
& \quad<\sum_{\bar{\gamma}: o(\bar{\gamma})=A}\left\langle n_{b}(\bar{\gamma})\right\rangle\left\{\sum_{\substack{\bar{\Gamma} \in \bar{\gamma} \\
x(\Gamma) \in V}}\left|\rho_{V, A}(\bar{\Gamma})-\rho_{A}(\bar{\gamma})\right|+\sum_{\bar{\Gamma} \in \bar{\gamma}: x(\Gamma) \in V \bar{\Gamma} \nsubseteq V} \rho_{V, A}(\bar{\Gamma})\right\} \\
& \quad<F_{1}(\beta)|\partial V| .
\end{aligned}
$$

As for the variance of $N_{A}(\cdot ; V)$, we have the following formula.

$$
\begin{aligned}
D_{V, A}\left(N_{A}\right)= & \sum_{\bar{\gamma}: o(\bar{\gamma})=A} D\left(n_{A}(\bar{\gamma})\right) \sum_{\bar{\Gamma} \in \bar{\gamma}} \rho_{V, A}(\bar{\Gamma}) \\
& +\sum_{\substack{\bar{\gamma}_{1} \neq \bar{\gamma}_{2} \\
o\left(\bar{\gamma}_{1}\right)=o\left(\bar{\gamma}_{2}\right)=A}}\left(\left|V\left(\gamma_{1}\right)\right|-\left\langle n_{A}\left(\bar{\gamma}_{1}\right)\right\rangle\right)\left(\left|V\left(\gamma_{2}\right)\right|-\left\langle n_{A}\left(\bar{\gamma}_{2}\right)\right\rangle\right) \\
& \times \sum_{\substack{\bar{\Gamma}_{1} \in \bar{\gamma}_{1} \\
\bar{\Gamma}_{2} \in \bar{\gamma}_{2}}}\left\{\rho_{V, A}\left(\bar{\Gamma}_{1}, \bar{\Gamma}_{2}\right)-\rho_{V, A}\left(\bar{\Gamma}_{1}\right) \rho_{V, A}\left(\bar{\Gamma}_{2}\right)\right\} \\
& +\sum_{\bar{\gamma}: o(\bar{\gamma})=A}\left(|V(\gamma)|-\left\langle n_{A}(\bar{\gamma})\right\rangle\right)^{2} \sum_{\bar{\Gamma} \in \bar{\gamma}} \rho_{V, A}(\bar{\Gamma})\left(1-\rho_{V, A}(\bar{\Gamma})\right) .
\end{aligned}
$$

By using Lemma 3.7 we have

$$
\begin{aligned}
& \left|D_{V, A}\left(N_{A}\right)\right| \\
& <\sum_{\substack{\bar{\gamma}_{1} \neq \bar{\gamma}_{2} \\
o\left(\bar{\gamma}_{1}\right)=o\left(\bar{\gamma}_{2}\right)=A}} c_{2}\left|\gamma_{1}\right|^{2}\left|\gamma_{2}\right|^{2} a\left(\bar{\gamma}_{1}\right) a\left(\bar{\gamma}_{2}\right) \cdot|V| \sum_{k=1}^{\infty}\left(\left|\gamma_{1}\right|+8 k\right)\left|\gamma_{2}\right| e^{-(3 / 2) \beta \varepsilon k} \\
& \quad+2 \sum_{\bar{\gamma}: o(\bar{\gamma})=A}|\gamma|^{2} e^{-\beta E(\bar{\gamma})} \cdot|V| \\
& <c_{2}|V|\left\{\sum_{\bar{\gamma}: o(\bar{\gamma})=A}|\gamma|^{3} a(\bar{\gamma})\right\}^{2} \sum_{k=1}^{\infty}(1+2 k) e^{-(3 / 2) \beta \varepsilon k} \\
& \quad+2|V| \sum_{\bar{\gamma}: o(\bar{\gamma})=A}|\gamma|^{2} e^{-\beta E(\bar{\gamma})} \\
& <F_{2}(\beta)|V|,
\end{aligned}
$$


where $F_{2}(\beta) / \exp (-3 \beta \varepsilon) \downarrow 0$ exponentially as $\beta \rightarrow \infty$. When $b=A$ we have

$$
\begin{aligned}
& D_{V, A}\left(N_{b}\right)=\sum_{\bar{\gamma}: o(\bar{\gamma})=A} D\left(n_{b}(\bar{\gamma})\right) \sum_{\bar{\Gamma} \in \bar{\gamma}} \rho_{V, A}(\bar{\Gamma}) \\
& \quad+\sum_{\substack{\bar{\gamma}_{1} \neq \bar{\gamma}_{2} \\
o\left(\bar{\gamma}_{1}\right)=o\left(\bar{\gamma}_{2}\right)=A}}\left\langle n_{b}\left(\bar{\gamma}_{1}\right)\right\rangle\left\langle n_{b}\left(\bar{\gamma}_{2}\right)\right\rangle \sum_{\substack{\bar{\Gamma}_{1} \in \bar{\gamma}_{1} \\
\bar{\Gamma}_{2} \bar{\gamma}_{2}}}\left\{\rho_{V, A}\left(\bar{\Gamma}_{1}, \bar{\Gamma}_{2}\right)-\rho_{V, A}\left(\bar{\Gamma}_{1}\right) \rho_{V, A}\left(\bar{\Gamma}_{2}\right)\right\} \\
& +\sum_{\bar{\gamma}: o(\bar{\gamma})=A}\left\langle n_{b}(\bar{\gamma})\right\rangle^{2} \sum_{\bar{\Gamma} \in \bar{\gamma}} \rho_{V, A}(\bar{\Gamma})\left(1-\rho_{V, A}(\bar{\Gamma})\right) .
\end{aligned}
$$

By the same way we have

$$
\left|D_{V, A}\left(N_{b}\right)\right|<F_{2}(\beta)|V| \text {. }
$$

We summurize the above estimates in the following.

Proposition 5.1. For sufficiently large $\beta$, we have

1) $\left|\left\langle N_{A}\right\rangle_{V, A}-\rho_{1}^{* *}\right| V||<F_{1}(\beta)|\partial V|$

2) $\left|\left\langle N_{b}\right\rangle_{V, A}-\rho_{2}^{*}\right| V||<F_{1}(\beta)|\partial V| \quad(b \neq A)$

3) $\left|D_{V, A}\left(N_{A}\right)\right|<F_{2}(\beta)|V|$

4) $\left|D_{V, A}\left(N_{b}\right)\right|<F_{2}(\beta)|V|$

where $F_{1}(\beta) \sim F_{2}(\beta)$ and $F_{1}(\beta) / \exp (-3 \beta \varepsilon) \downarrow 0$ exponentially as $\beta \rightarrow \infty$.

\section{§6. The Estimates of $\mathbb{P}_{V, A}\left(\mathbb{N}_{V}^{(1)}\right)$ and $\mathbb{P}_{V, A}\left(\mathbb{N}_{V}^{(2)}\right)$ from $\mathbb{R}$ elow}

From now on we restrict our attention to the model composed of four types of particles satisfying the conditions (c.1) and (D.1).

By using the properties of correlation functions we estimate the probabilities $P_{V, A}\left(N_{V}^{(1)}\right)$ and $P_{V, A}\left(N_{V}^{(2)}\right)$ from below. Let $\Gamma_{1}$ and $\Gamma_{2}$ be the two squares with the breadth of $r_{B}^{1 / 2}|V|^{1 / 2}$ and $r_{D}^{1 / 2}|V|^{1 / 2}$ arranged as in Figure 3. Put $\bar{\Gamma}_{1}=\left(\Gamma_{1}, A, B\right), \bar{\Gamma}_{2}=\left(\Gamma_{2}, A, D\right)$ and $\mathscr{M}=\left\{\xi \in \Omega_{V, A}: \bar{\Gamma}_{1}, \bar{\Gamma}_{2} \in \xi_{\text {out }}\right\}$. Let $P_{\mathscr{A}}(\cdot)$ be the conditional probability $P_{V, A}(\cdot \mid \mathscr{M})$, then

$$
P_{V, A}\left(N_{V}^{(1)}\right) \geqq P_{V, A}(\mathscr{M}) P_{\mathscr{M}}\left(N_{V}^{(1)}\right) \text {. }
$$

We first estimate the probability $P_{\mathscr{M}}\left(N_{V}^{(1)}\right)$ from below. Put $V_{2}=\theta\left(\Gamma_{1}\right)$, $V_{3}=\theta\left(\Gamma_{2}\right)$, and $V_{1}=V \backslash\left(w\left(\Gamma_{1}\right) \cup w\left(\Gamma_{2}\right)\right)$. Let $\mathscr{A}_{1}$ be the subset of $\mathscr{M}$ given by

$$
\begin{aligned}
\mathscr{A}_{1}=\{\xi \in \mathscr{M} ; & \left|N_{A}\left(\xi ; V_{1}\right)-\rho_{1}^{* *}\right| V_{1}||<F_{2}(\beta)^{1 / 3}\left|V_{1}\right|^{1 / 2}, \\
& \left|N_{B}\left(\xi ; V_{2}\right)-\rho_{1}^{* *}\right| V_{2}||<F_{2}(\beta)^{1 / 3}\left|V_{2}\right|^{1 / 2}, \text { and } \\
& \left.\left|N_{D}\left(\xi ; V_{3}\right)-\rho_{1}^{* *}\right| V_{3}||<F_{2}(\beta)^{1 / 3}\left|V_{3}\right|^{1 / 2}\right\} .
\end{aligned}
$$

It is easily seen that $\mathscr{A}_{1} \subset N_{V}^{(1)}$ for sufficiently large $\beta$ and $V$. Therefore,

$$
P_{\mathscr{M}}\left(N_{V}^{(1)}\right) \geqq P_{\mathscr{M}}\left(\mathscr{A}_{1}\right) \text {. }
$$


By using the Chevyshev's inequality, we have

$$
\begin{aligned}
& P_{V_{1}, A}\left(\left|N_{A}\left(\xi: V_{1}\right)-\rho_{1}^{* *}\right| V_{1}||<F_{2}(\beta)^{1 / 3}\left|V_{1}\right|\right) \\
& \quad>P_{V_{1}, A}\left(\left|N_{A}\left(\xi: V_{1}\right)-\left\langle N_{A}\right\rangle_{V_{1}, A}\right|<\frac{1}{2} F_{2}(\beta)^{1 / 3}\left|V_{1}\right|\right) \\
& \quad>1-4 F_{2}(\beta)^{1 / 3} .
\end{aligned}
$$

Similarly, we have

$$
\begin{aligned}
& P_{V_{2}, B}\left(\left|N_{B}\left(\xi: V_{2}\right)-\rho_{1}^{* *}\right| V_{2}||<F_{2}(\beta)^{1 / 3}\left|V_{2}\right|\right)>1-4 F_{2}(\beta)^{1 / 3} \\
& P_{V_{3}, D}\left(\left|N_{D}\left(\xi: V_{3}\right)-\rho_{1}^{* *}\right| V_{3}||<F_{2}(\beta)^{1 / 3}\left|V_{3}\right|\right)>1-4 F_{2}(\beta)^{1 / 3} .
\end{aligned}
$$

Hence, $P_{\mathscr{M}}\left(N_{V}^{(1)}\right)>9 / 10$ for sufficiently large $\beta$.

Next we estimate the probability $P_{V, A}(\mathscr{M})$ from below. We can express $P_{V, A}(\mathscr{M})$ as follows.

$$
P_{V, A}(\mathscr{M})=\frac{1}{Z_{V, A}} e^{-\beta\left(E\left(\bar{\Gamma}_{1}\right)+E\left(\bar{\Gamma}_{2}\right)\right)} \cdot Z_{V_{1}, A} \cdot Z_{V_{2}, B} \cdot Z_{V_{3}, D} \cdot
$$

Taking into account the fact that

$$
\frac{\partial \ln Z_{V, a}}{\partial E(\bar{\gamma})}=-\beta \sum_{\substack{\bar{\Gamma} \in \bar{\gamma} \\ \Gamma \subset V, A}} \tau_{V, A}(\bar{\Gamma})
$$

and putting $E_{t}(\bar{\gamma})=t E(\bar{\gamma})$, we have from $(6.4)$

$$
\begin{aligned}
& \ln P_{V, A}(\mathscr{M})=-4 \beta \varepsilon_{1}\left(r_{B}^{1 / 2}+r_{D}^{1 / 2}\right)|V|^{1 / 2}+\beta_{o}\left(|V|^{1 / 2}\right) \\
& -\int_{1}^{\infty} d t\left\{\frac{d}{d t} \ln Z_{V_{1}, A}(t)+\frac{d}{d t} \ln Z_{V_{2}, A}(t)\right. \\
& \left.+\frac{d}{d t} \ln Z_{V_{3}, A}(t)-\frac{d}{d t} \ln Z_{V, A}(t)\right\} \\
& =-4 \beta \varepsilon_{1}\left(r_{B}^{1 / 2}+r_{D}^{1 / 2}\right)|V|^{1 / 2}+\beta_{o}\left(|V|^{1 / 2}\right) \\
& +\beta \sum_{\bar{\gamma}} E(\bar{\gamma}) \int_{1}^{\infty} d t\left\{\sum_{\bar{\Gamma} \in \bar{\gamma} \Gamma \subset V_{1}} \tau_{V_{1}, A, t}(\bar{\Gamma})\right. \\
& \left.+\sum_{\substack{\bar{T} \in \overline{\bar{\gamma}} \\
\Gamma \subset V_{2}}} \tau_{V_{2} A, t}(\bar{\Gamma})+\sum_{\substack{\bar{T} \in \bar{\gamma} \\
\Gamma \subset V_{3}}} \tau_{V_{3}, A, t}(\bar{\Gamma})-\sum_{\substack{\bar{\Gamma} \in \bar{\gamma} \\
\Gamma \subset V}} \tau_{V, A, t}(\bar{\Gamma})\right\} .
\end{aligned}
$$

Here we have used the fact that $Z_{V, a}$ is independent of a from the symmetry of the interactions.

We first estimate the second term in the right hand side of (6.6) as follows by employing the properties of correlation functions obtained in Section 3,

$$
\begin{aligned}
& \left|\sum_{\substack{\bar{T} \in \bar{\gamma} \\
\Gamma \subset V_{1}}} \tau_{V_{1}, A, t}(\bar{\Gamma})+\sum_{\substack{\bar{\Gamma} \in \bar{\gamma} \\
\Gamma \subset V_{2}}} \tau_{V_{2}, A, t}(\bar{\Gamma})+\sum_{\substack{\bar{\Gamma} \in \bar{\gamma} \\
\Gamma \subset V_{3}}} \tau_{V_{3}, A, t}(\bar{\Gamma})-\sum_{\substack{\bar{\Gamma} \in \bar{\gamma} \\
\Gamma \subset V}} \tau_{V, A, t}(\bar{\Gamma})\right| \\
& \quad<\sum_{\substack{\bar{\Gamma} \in \overline{\bar{\gamma}} \\
\Gamma \subset V_{1}}}\left|\tau_{V_{1}, A, t}(\bar{\Gamma})-\tau_{V, A, t}(\bar{\Gamma})\right|+\sum_{\substack{\bar{\Gamma} \in \bar{\gamma} \\
\Gamma \subset V_{2}}}\left|\tau_{V_{2}, A, t}(\bar{\Gamma})-\tau_{V, A, t}(\bar{\Gamma})\right|
\end{aligned}
$$




$$
\begin{aligned}
& \quad+\sum_{\substack{\bar{\Gamma} \in \bar{\gamma} \\
\Gamma \subset V_{3}}}\left|\tau_{V_{3}, A, t}(\bar{\Gamma})-\tau_{V, A, t}(\bar{\Gamma})\right|+\sum_{\substack{\Gamma \in \bar{\gamma} \\
\Gamma \subset V \\
\Gamma \text { intersect } \Gamma_{1}}} \tau_{V, A, t}(\bar{\Gamma})+\sum_{\substack{\bar{\Gamma} \in \bar{\gamma} \\
\Gamma \text { in } \\
\Gamma \text { intersect } \Gamma_{2}}} \tau_{V, A, t}(\bar{\Gamma}) \\
& <\text { const. }|V|^{1 / 2} \exp (-\beta E(\bar{\gamma}) t) .
\end{aligned}
$$

If we fix $\beta$ sufficiently large, then the following estimate holds for sufficiently large $V$,

$$
\left.\left.\left|\ln P_{V, A}(\mathscr{M})+4 \beta \varepsilon_{1}\left(r_{B}^{1 / 2}+r_{D}^{1 / 2}\right)\right| V\right|^{1 / 2}|<m(\beta)| V\right|^{1 / 2}
$$

where $m(\beta) / \exp \left(-3 \beta \varepsilon_{1}\right) \downarrow 0$ exponentially as $\beta \rightarrow \infty$.

We summurize the above estimate in the following proposition.

Proposition 6.1. If we fix $\beta$ sufficiently large, then the following estimate holds for sufficiently large $V$,

$$
P_{V, A}\left(N_{V}^{(1)}\right)>\frac{9}{10} \exp \left\{-4 \beta \varepsilon_{1}\left(r_{B}^{1 / 2}+r_{D}^{1 / 2}\right)|V|^{1 / 2}-m(\beta)|V|^{1 / 2}\right\}
$$

where $m(\beta) / \exp \left(-3 \beta \varepsilon_{1}\right) \downarrow 0$ exponentially as $\beta \rightarrow \infty$.

Next we estimate the probability $P_{V, A}\left(N_{V}^{(2)}\right)$. Let $\Gamma_{1}$ and $\Gamma_{2}$ be the two squares with the breadth of $\left(r_{B}+r_{C}\right)^{1 / 2}|V|^{1 / 2}$ and $r_{C}^{1 / 2}|V|^{1 / 2}$ arranged as in Figure 3. Put $\bar{\Gamma}_{1}=\left(\Gamma_{1}, A, B\right), \bar{\Gamma}_{2}=\left(\Gamma_{2}, B, C\right), V_{3}=\theta\left(\Gamma_{2}\right), V_{2}=\theta\left(\Gamma_{1}\right) \mid w\left(\Gamma_{2}\right)$ and $V_{1}=V \backslash w\left(\Gamma_{1}\right)$. Let $\mathscr{N}$ be the set of all configurations such that $\bar{\Gamma}_{1} \in \xi_{\text {out }}$, $\bar{\Gamma}_{2} \in \xi$, and every $\bar{\Gamma} \in \xi \backslash \bar{\Gamma}_{1}$ does not surround $\Gamma_{2}$.

(1)

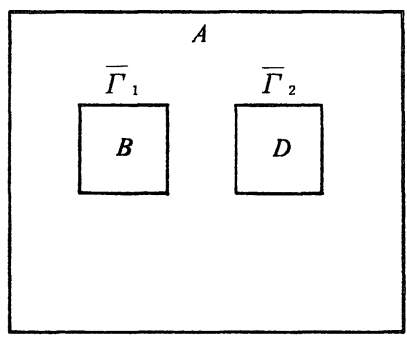

(2)

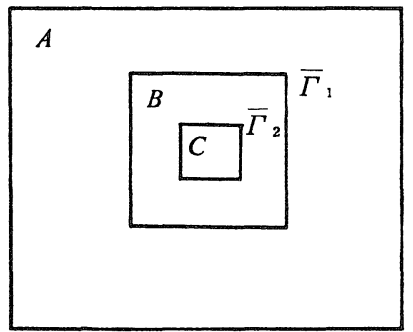

Figure 3.

Let $P_{\mathscr{S}}(\cdot)$ be the conditional probability $P_{V, A}(\cdot \mid \mathscr{N})$, then

$$
P_{V, A}\left(N_{V}^{(2)}\right) \geqq P_{V, A}(\mathscr{N}) P_{\mathscr{N}}\left(N_{V}^{(2)}\right) .
$$

Let $\mathscr{A}_{2}$ be the set of $\mathscr{N}$ given by

$$
\begin{aligned}
\mathscr{A}_{2}=\{\xi \in N ; & \left|N_{A}\left(\xi ; V_{1}\right)-\rho_{1}^{* *}\right| V_{1}||<F_{2}(\beta)^{1 / 3}\left|V_{1}\right|^{1 / 2}, \\
& \left|N_{B}\left(\xi ; V_{2}\right)-\rho_{1}^{* *}\right| V_{2}||<F_{2}(\beta)^{1 / 3}\left|V_{2}\right|^{1 / 2}, \\
& \left.\left|N_{C}\left(\xi ; V_{3}\right)-\rho_{1}^{* *}\right| V_{3}||<F_{2}(\beta)^{1 / 3}\left|V_{3}\right|^{1 / 2}\right\}
\end{aligned}
$$


then $\mathscr{A}_{2} \subset N_{V}^{(2)}$ for every sufficiently large and $V$. Theorefore we have

$$
\begin{aligned}
P_{\mathscr{N}}( & \left.N_{V}^{(2)}\right) \geqq P_{\mathscr{N}}\left(\mathscr{A}_{2}\right) \\
= & P_{V_{1}, A}\left(\left|N_{A}\left(\xi ; V_{1}\right)-\rho_{1}^{* *}\right| V_{1}||<F_{2}(\beta)^{1 / 3}\left|V_{1}\right|^{1 / 2}\right) \\
& \times P_{V_{2}, B}\left(\left|N_{B}\left(\xi ; V_{2}\right)-\rho_{1}^{* *}\right| V_{2}||<F_{2}(\beta)^{1 / 3}\left|V_{2}\right|^{1 / 2}\right) \\
& \times P_{V_{3}, C}\left(\left|N_{C}\left(\xi ; V_{3}\right)-\rho_{1}^{* *}\right| V_{3}||<F_{2}(\beta)^{1 / 3}\left|V_{3}\right|^{1 / 2}\right) .
\end{aligned}
$$

In the same way as we obtained that $P_{\mathscr{A}}\left(N_{V}^{(1)}\right) \geqq 9 / 10$, we have

$$
P_{\mathscr{S}}\left(N_{V}^{(2)}\right) \geqq 9 / 10 \quad \text { for sufficiently large } \beta \text {. }
$$

We also have the following estimate of $P_{V, A}(\mathcal{N})$ by the same way as we have obtained the estimate of $P_{V, A}(\mathscr{M})$,

$$
\left.\left.\left|\ln P_{V, A}(\mathscr{N})+4 \beta \varepsilon_{1}\left\{\left(r_{B}+r_{C}\right)^{1 / 2}+r_{C}^{1 / 2}\right\}\right| V\right|^{1 / 2}|<m(\beta)| V\right|^{1 / 2}
$$

where $m(\beta) / \exp \left(-3 \beta \varepsilon_{1}\right) \downarrow 0$ exponentially as $\beta \rightarrow \infty$.

Hence we have the following.

Proposition 6.2. If we fix $\beta$ sufficiently large, the the following estimate holds for sufficiently large $V$,

$$
P_{V, A}\left(N_{V}^{(2)}\right)>\frac{9}{10} \exp \left[\left\{-4 \beta \varepsilon_{1}\left(\left(r_{B}+r_{C}\right)^{1 / 2}+r_{C}^{1 / 2}\right)-m(\beta)\right\}|V|^{1 / 2}\right]
$$

where $m(\beta) / \exp \left(-3 \beta \varepsilon_{1}\right) \downarrow 0$ exponentially as $\beta \rightarrow \infty$.

\section{§7. Proof of Theorems}

In this section we give the proof of theorems by estimating the length of phase boundaries and the number of particles in each phase.

The following proposition performs an important part of the proof.

Proposition 7.1. For sufficiently large $\beta$ and $V$, we have

1) $\quad P_{N_{V}^{(i)}}\left(\left|N_{a}\left(\xi: \theta_{a}\right)-\rho_{1}^{* * *}\right| \theta_{a}||>t\left|\theta_{a}\right|^{3 / 4},\left|\theta_{a}\right|>k|V|\right)$

$$
<C \exp \left[-\left(q(\beta) t^{2} k^{1 / 2}-8 \beta \varepsilon_{1}\right)|V|^{1 / 2}\right]
$$

for any $t>0$ and $k>0(i=1,2)$.

2) $P_{N_{V}^{(i)}}\left(\left|N_{a}\left(\xi: \bigcup_{b \neq a} \theta_{b}\right)-\rho_{1}^{*}\left(\sum_{b \neq a}\left|\theta_{b}\right|\right)\right|>t\left(\sum_{b \neq a}\left|\theta_{b}\right|\right)^{3 / 4}, \sum_{b \neq a}\left|\theta_{b}\right|>k|V|\right)$

$$
<C \exp \left[-\left(q(\beta) t^{2} k^{1 / 2}-8 \beta \varepsilon_{1}\right)|V|^{1 / 2}\right]
$$

for any $t>0$ and $k>0(i=1,2)$, where $\theta_{a}$ is "a-phase", $N_{a}\left(\cdot ; \theta_{a}\right)$ is the number of $a$-particles in $\theta_{a}$, and $q(\beta) / \exp \left(2 \beta \varepsilon_{1}\right) \uparrow \infty$ exponentially as $\beta \rightarrow \infty$. 
The proof of this proposition is decomposed into several steps. We first estimate the probability of the following event $\mathrm{m}_{V, c_{0}}$ with respect to $P_{V, A}(\cdot)$,

$$
\mathfrak{m}_{V, c_{0}}=\left\{\xi \in \Omega_{V, A} ; \sup _{\bar{\Gamma} \in \xi_{\text {out }}}|\Gamma|<c_{0} \ln |V|\right\}
$$

Lemma 7.1. For every sufficiently large $\beta$, the following estimate is satisfied:

$$
P_{V, A}\left(\mathrm{~m}_{V, c_{0}}\right) \geqq 1-\frac{d_{1}}{|V|^{(3 / 4) \beta \varepsilon c_{0}-1}}
$$

Proof. From Lemmas 3.1 and 4.1, we have

$$
\begin{aligned}
P_{V, A}\left(\mathrm{~m}_{V, c_{0}}^{c}\right) & \leqq \sum_{\substack{\bar{\Gamma}: 0(\bar{\Gamma})=A \\
\Gamma \subset V,|\Gamma| \geqq c_{0} \ln |V|}} \rho_{V, A}(\bar{\Gamma}) \\
& \leqq|V| \sum_{\substack{\bar{\Gamma}: \Gamma \ni 0 \\
|\Gamma| \geqq c_{0} \ln |V|}} \exp (-\beta E(\bar{\Gamma}))<\frac{d_{1}}{|V|^{(3 / 4) \beta \varepsilon c_{0}-1}} .
\end{aligned}
$$

Let $\Omega_{V, A, c_{0}}$ be the set of all families of type $A c_{0}$-small contours $\xi=\left(\bar{\Gamma}_{1}, \ldots, \bar{\Gamma}_{s}\right)$ such that $w\left(\Gamma_{1}\right), \ldots, w\left(\Gamma_{s}\right)$ are mutually disjoint.

We introduce the probability measure $P_{V, A, c_{0}}(\cdot)$ as follows

$$
P_{V, A, c_{0}}(\xi)=\frac{1}{Z_{V, A, c_{0}}} \prod_{\Gamma \in \xi} \exp (-\beta E(\bar{\Gamma})) Z_{\theta(\Gamma), i(\bar{\Gamma})}
$$

where

$$
Z_{\theta, a}=\sum_{\xi \in \Omega_{\theta}, a} \exp \left(-\beta U_{\theta}(\xi)\right) .
$$

Let $\theta$ be the set of $V$ satisfying $|\theta|>k|V|$. We denote the number of outer contours $\Gamma$ which belong to $\bar{\gamma}$ under the configuration $\xi$ by $N(\bar{\gamma} ; \xi)$. We now consider the asymptotic behavior of $N_{a}(\xi ; \theta)$ as $|V| \rightarrow \infty$ under the condition that all outer contours in $\theta$ are $c_{0}$-small. Put

$$
u_{\lambda}(\xi ; \theta)=\frac{1}{|\theta|^{3 / 4}} \sum_{\bar{\gamma}: c^{-}-\mathrm{mall}}\left|N(\bar{\gamma} ; \xi)-\sum_{\substack{\bar{\Gamma} \in \bar{\gamma} \\ \Gamma \subset \theta}} \rho_{\theta, A}(\bar{\Gamma})\right| e^{\lambda|\gamma|} \cdot|\gamma|
$$

where $\lambda=1 / 5 c_{0}$ and $c_{0}<1 / 2 \ln 3$. Note that $u_{\lambda}(\xi ; \theta)$ is determined by $\xi_{\text {out }}$. As for the estimate of $u_{\lambda}(\xi ; \gamma)$, the following lemma is satisfied.

Lemma 7.2. For sufficiently large $\beta$, we have

$$
\begin{aligned}
& P_{\theta, A}\left(u_{\lambda}(\xi ; \theta)>T, N(\gamma ; \xi)=0 \text { for all } c_{0} \text {-large } \bar{\gamma}\right) \\
& \quad<2 \exp \left[-c(\beta) T^{2} k^{1 / 2}|V|^{1 / 2}\right]
\end{aligned}
$$

where $c(\beta) / \exp \left(2 \beta \varepsilon_{1}\right) \uparrow \infty$ exponentially as $\beta \rightarrow \infty$. 
The proof of this lemma is given in the appendix.

From Lemma 7.1 we have

$$
Z_{V, A, c_{0}} / Z_{V, A}>9 / 10 \quad \text { for sufficiently large } \beta \text {. }
$$

Taking into account (7.2), we have the following lemma from Lemma 7.2.

Lemma 7.3. For sufficiently large $\beta$ and $V$, we have

$$
P_{\theta, A, c_{0}}\left(u_{\lambda}(\xi ; \theta)>T\right)<\frac{20}{9} \exp \left(-c(\beta) T^{2} k^{1 / 2}|V|^{1 / 2}\right) .
$$

We prepare the following standard argument of probability theory.

Lemma 7.4. Let $X_{1}, \ldots, X_{n}$ be the sequence of independent random variables and assume that $R_{i}(\alpha)=\left\langle\exp \left(x_{i} \alpha\right)\right\rangle$ exists for each $i$ and $\alpha$. Further we assume that

$$
0<\frac{\partial^{2} \ln R_{i}(\alpha)}{\partial \alpha^{2}}<c_{i}
$$

Then we have,

$$
\operatorname{Prob}\left(\left|X_{1}+\cdots+X_{n}-s\right|>x\right)<2 \exp \left(-x^{2} / 2 c\right),
$$

where $c=\sum_{i=1}^{n} c_{i}$ and $s=\sum_{i=1}^{n}\left\langle X_{i}\right\rangle$.

We take a family of outer contours $\left(\bar{\Gamma}_{1}, \ldots, \bar{\Gamma}_{s}\right)$ satisfying $u_{\lambda}\left(\left\{\bar{\Gamma}_{1}, \ldots, \bar{\Gamma}_{s}\right\} ; \theta\right)$ $<T$, and denote the conditional probability measure under the condition that the totality of outer contours is $\left(\bar{\Gamma}_{1}, \ldots, \bar{\Gamma}_{s}\right)$ by $P_{\left(\bar{\Gamma}_{1}, \ldots, \bar{\Gamma}_{s}\right)}$. By using Lemma 7.4 , we have

$$
\begin{aligned}
& P_{\left(\bar{\Gamma}_{1}, \ldots, \bar{\Gamma}_{s}\right)}\left(\left|N_{A}(\xi ; \theta)-<N_{A}(\xi ; \theta)>_{\left(\bar{\Gamma}_{1}, \ldots, \bar{\Gamma}_{s}\right)}\right|>t|\theta|^{3 / 4}\right) \\
& \quad \leqq 2 \exp \left(-\frac{t^{2}|\theta|^{3 / 2}}{2 \sum_{\bar{\gamma}: c_{0} \text {-small }} N\left(\bar{\gamma} ; \bar{\Gamma}_{1}, \ldots, \bar{\Gamma}_{s}\right)|V(\gamma)|^{2}}\right) .
\end{aligned}
$$

As $u_{\lambda}\left(\bar{\Gamma}_{1}, \ldots, \bar{\Gamma}_{s} ; \theta\right)<T$, we have

$$
\begin{aligned}
& \sum_{\bar{\gamma}: c 0^{-s m a l l}} N\left(\bar{\gamma} ; \bar{\Gamma}_{1}, \ldots, \bar{\Gamma}_{s}\right)|v(\gamma)|^{2} \\
& \quad<\sum_{\bar{\gamma}: c_{0} \text {-small }}\left|N\left(\bar{\gamma} ; \bar{\Gamma}_{1}, \ldots, \bar{\Gamma}_{s}\right)-\sum_{\substack{\bar{\Gamma} \in \bar{\gamma} \\
\Gamma \subset \theta}} \rho_{\theta, A}(\bar{\Gamma})\right||v(\gamma)|^{2} \\
& \quad+\sum_{\substack{\bar{\Gamma}: C_{0} \text {-small } \\
\Gamma \subset \theta}} \rho_{\theta, A}(\bar{\Gamma})|v(\gamma)|^{2} \\
& \quad<\operatorname{Max}_{k \geqq 4} k^{3} e^{-\lambda k}|\theta|^{3 / 4} u_{\lambda}\left(\bar{\Gamma}_{1}, \ldots, \bar{\Gamma}_{s} ; \theta\right)+\sum_{\bar{\Gamma}: c_{0} \text {-small }} e^{-\beta E(\bar{\Gamma})}|\Gamma|^{4} \\
& \quad<\operatorname{Max}_{k \geqq 4} k^{3} e^{-\lambda k} \cdot T \cdot|\theta|^{3 / 4}+|\theta| \sum_{\bar{\gamma}: c_{0} \text {-small }}|\gamma|^{4} e^{-\beta E(\bar{\gamma})} \\
& <s(\beta)|\theta|
\end{aligned}
$$


where $s(\beta) / \exp \left(-3 \beta \varepsilon_{1}\right) \downarrow 0$ exponentially as $\beta \rightarrow \infty$.

From (7.4) and (7.5), we have

$$
\begin{aligned}
& P_{\left(\bar{\Gamma}_{1}, \ldots . \bar{\Gamma}_{s}\right)}\left(\left|N_{A}(\xi ; \theta)-<N_{A}(\zeta ; \theta)>_{\left(\bar{\Gamma}_{1}, \ldots, \bar{\Gamma}_{s}\right)}\right|>t|\theta|^{3 / 4}\right) \\
& \quad \leqq 2 \exp \left(-\left(t^{2} / 2 s(\beta)\right) \cdot k^{1 / 2} \cdot|V|^{1 / 2}\right) .
\end{aligned}
$$

From the definition of $u_{\lambda}\left(\bar{\Gamma}_{1}, \ldots, \bar{\Gamma}_{s} ;\right)$, we have

$$
\begin{aligned}
& \left|\left\langle N_{A}\right\rangle_{\left(\bar{\Gamma}_{1}, \ldots, \bar{\Gamma}_{s}\right)}-\left\langle N_{A}\right\rangle_{\theta, A . C_{0}}\right| \\
& \quad<\sum_{\bar{\gamma}: c_{0} \text {-small }}\left|N\left(\bar{\gamma}: \bar{\Gamma}_{1}, \ldots, \bar{\Gamma}_{s}\right)-\sum_{\bar{\Gamma} \in \bar{\gamma}} \rho_{\theta, A}(\bar{\Gamma})\right||\gamma|^{2} \\
& \quad+\left(Z_{V, A} / Z_{V, A, C_{0}}-1\right) \sum_{\bar{\Gamma}: c_{0} \text {-smal1 }} \rho_{\theta, A}(\bar{\Gamma})|\Gamma|^{2} \\
& \quad<M_{\lambda} u_{\lambda}\left(\bar{\Gamma}_{1}, \ldots, \bar{\Gamma}_{s}: \theta\right)|\theta|^{3 / 4}+d_{1} k|V| /|V|^{3 / 4 \beta \varepsilon c_{0}-1}-d_{1} \cdot \sum_{\bar{\gamma}: c_{0} \text {-small }} e^{-\beta E(\bar{\gamma})|\gamma|^{2}} \\
& \quad<\frac{11}{10} T M_{\lambda}|\theta|^{3 / 4} .
\end{aligned}
$$

For a given $t>0$, we choose the number $T>0$ satisfying $t>22 / 10 T M_{\lambda}$ and $T>1 / 3 t$. Then we have

$$
\begin{aligned}
& P_{\theta, A, C_{0}}\left(\left|N_{A}(\xi ; \theta)-\left\langle N_{A}\right\rangle_{\theta, A, C_{0}}\right|>t|\theta|^{3 / 4}, u_{\lambda}(\xi ; \theta)<T\right) \\
& \quad<2 \exp \left(-\left(t^{2} / 8 s(\beta)\right) \cdot k^{1 / 2}|V|^{1 / 2}\right) .
\end{aligned}
$$

From (7.3) and (7.8) we have

$$
\begin{aligned}
& P_{0 . A . c_{0}}\left(\left|N_{A}(\xi ; \theta)-\left\langle N_{A}\right\rangle_{\theta, A, c_{0}}\right|>t|\theta|^{3 / 4}\right) \\
& \quad<20 / 9 \exp \left(-c(\beta) T^{2} k^{1 / 2}|V|^{1 / 2}\right)+2 \exp \left(-\left(t^{2} / 8 s(\beta)\right) k^{1 / 2}|V|^{1 / 2}\right) \\
& \quad<\text { const. } \exp \left(-q^{\prime}(\beta) t^{2} k^{1 / 2}|V|^{1 / 2}\right)
\end{aligned}
$$

where $q^{\prime}(\beta) / \exp \left(2 \beta \varepsilon_{1}\right) \uparrow \infty$ as $\beta \rightarrow \infty$.

Taking into account the estimate

$$
\left|\left\langle N_{A}\right\rangle_{\theta, A, c_{0}}-\rho_{1}^{* *}\right| \theta||<h(\beta) k^{1 / 2}|V|^{1 / 2},\left(h(\beta) / \exp \left(-3 \beta \varepsilon_{1}\right) \downarrow 0\right)
$$

we have the following lemma from (7.9).

Lemma 7.5. For sufficiently large $\beta$ and $V$, we have

$$
\begin{aligned}
& P_{\theta, A, c_{0}}\left(\left.\left|N_{A}(\xi ; \theta)-\rho_{1}^{* *}\right| \theta|>t| \theta\right|^{3 / 4}\right) \\
& \quad<c \exp \left(-q(\beta) t^{2} k^{1 / 2}|V|^{1 / 2}\right)
\end{aligned}
$$

where $q(\beta) / \exp \left(2 \beta \varepsilon_{1}\right) \uparrow \infty$ exponentially as $\beta \rightarrow \infty$.

From Propositions 6.1, 6.2, and Lemma 7.5, Proposition 7.1 is easily proved.

Next we shall estimate the length of the totality of phase boundaries $\Lambda$. Let $M_{T, h}$ be the set of all configurations such that $\Lambda$ is composed of $k$ com- 
ponents and $|\Lambda|=T$. As the length of each phase boundary is greater than $c_{0} \ln |V|, k$ must be smaller than $T / c_{0} \ln |V|$.

By using the similar estimates stated in Sections 3 and 4, we have

$$
P_{V, A}\left(M_{T, k}\right)<4^{2} k 2^{T} 3^{T} \exp \left(-\beta \varepsilon_{1} T-\beta o(T)\right)|V|^{k}
$$

Put $M_{T}=\{\xi ;|\Lambda|=T\}$. From (7.12), we have

$$
\begin{aligned}
P_{V, A}\left(M_{T}\right) & <4^{2} 6^{T} \exp \left(-\beta \varepsilon_{1} T-\beta o(T)\right) \\
& <4^{2} \exp \left(-\left(\beta \varepsilon_{1}-\ln 6\right) T\right) e^{\left(2 / c_{0}\right) T} / \ln |V| \\
& <\exp \left(\left(-\beta \varepsilon_{1}+\ln 6+2 / c_{0}\right) T-\beta o(T)\right) .
\end{aligned}
$$

From Propositions 6.1, 6.2, and (7.13), we have the following Proposition.

Proposition 7.2. If we fix $\beta$ sufficiently large, then for each $t>0$ and every sufficiently large $V$, we have

1) $P_{N_{V}^{(1)}}\left(|\Lambda|>4\left(r_{B}^{1 / 2}+r_{D}^{1 / 2}\right)|V|^{1 / 2}+t|V|^{1 / 2}\right)$

$$
<\frac{20}{9} \exp \left[\left(-\left(\beta \varepsilon_{1}-u_{0}\right) t+8 u_{0}\right)|V|^{1 / 2}\right]
$$

2) $P_{N_{V}^{(2)}}\left(|\Lambda|>4\left(\left(r_{B}+r_{C}\right)^{1 / 2}+r_{c}^{1 / 2}\right)|V|^{1 / 2}+t|V|^{1 / 2}\right)$

$$
<\frac{20}{9} \exp \left[\left(-\left(\beta \varepsilon_{1}-u_{0}\right) t+8 u_{0}\right)|V|^{1 / 2}\right]
$$

where $u_{0}=\ln 6+2 / c_{0}$.

From this proposition we have

$$
\begin{aligned}
& |\Lambda|<4\left(r_{B}^{1 / 2}+r_{D}^{1 / 2}\right)|V|^{1 / 2}+k_{0}(\beta)|V|^{1 / 2} \quad \text { a.w. } P_{N_{V}^{(1)}} \text {-prob. } 1 \\
& |\Lambda|<4\left(\left(r_{B}+r_{C}\right)^{1 / 2}+r_{C}^{1 / 2}\right)|V|^{1 / 2}+k_{0}(\beta)|V|^{1 / 2} \quad \text { a.w. } P_{N_{V}^{(2)}} \text {-prob.1 }
\end{aligned}
$$

where $k_{0}(\beta)=\left(8 u_{0}+1\right) /\left(\beta \varepsilon_{1}-u_{0}\right)$.

First we prove Theorem 2.1. Let $r(\beta)$ be the function of $\beta$ satisfying $r(\beta) \sim \exp \left(-3 \beta \varepsilon_{1}\right)$. If $\left|\theta_{a}\right|>(1+r(\beta)) r_{a}|V|(a=B$ or $D)$, then from Proposition 7.1 we have the following estimate

$$
\begin{aligned}
N_{a}\left(\xi ; \theta_{a}\right) & >\rho_{1}^{* *} r_{a}(1+r(\beta))|V|-r(\beta)^{1 / 4}|V|^{3 / 4} \\
& >r_{a}|V|+1 / 2 r_{a} r(\beta)|V| \quad \text { a.w. } P_{N_{V}^{(1)}} \text {-prob. } 1
\end{aligned}
$$

for sufficiently large $\beta$.

On the other hand,

$$
N_{a}(\xi ; V)<r_{a}|V|+\rho_{1}^{*}|V| \quad \text { for any } \xi \in N_{V}^{(1)} .
$$

Because $1 / 2 r_{a} r(\beta)>\rho_{1}^{*}(\beta)$ for every sufficiently large $\beta,(7.16)$ is inconsistent with (7.17). Hence, 


$$
\left|\theta_{a}\right|<(1+r(\beta)) r_{a}|V| \quad \text { a.w. } P_{N_{V}^{(1)}} \text {-prob. } 1(a=B \text { or } D)
$$

If $\left|\theta_{a}\right|<(1-r(\beta)) r_{a}|V|$, then

$$
\sum_{b \neq a}\left|\theta_{b}\right|>\left(1-r_{a}(1-r(\beta))|V| .\right.
$$

From Proposition 7.1, we have

$$
N_{a}\left(\xi ; \underset{b \neq a}{\cup} \theta_{b}\right)<2 \rho_{1}^{*}|V| .
$$

Hence we have

$$
\begin{aligned}
N_{a}(\xi ; V) & <(1-r(\beta)) r_{a}|V|+2 \rho_{1}^{*}|V| \\
& <r_{a}|V|-1 / 2 r_{a} r(\beta)|V|
\end{aligned}
$$

for every sufficiently large $\beta$.

On the other hand,

$$
N_{a}(\xi ; V)>r_{a}|V|-\rho_{1}^{*}|V| \quad \text { for any } \xi \in N_{V}^{(1)} .
$$

Because $(1 / 2) r_{a} r(\beta)>\rho_{1}^{*}(\beta)$ for every sufficiently large $\beta$, (7.19) is inconsistent with (7.20). Therefore, we have

$$
\left|\theta_{a}\right|>(1-r(\beta)) r_{a}|V| \quad \text { a.w. } P_{N_{V}^{(1)}} \text {-prob. } 1 .
$$

From (7.18) and (7.21), we have the following lemma.

Lemma 7.6. For sufficiently large $\beta$, we have

$$
\left\|\theta_{a}\left|-r_{a}\right| V\right\|<r(\beta)|V| \quad \text { a.w. } P_{N_{V}^{(1)}}-\text { prob. } 1,
$$

where $r(\beta) \sim \exp \left(-3 \beta \varepsilon_{1}\right)$.

Let $\theta_{a}^{\max }$ be the largest connected component of $\theta_{a}$. Put $\theta_{a}^{\mathrm{rem}}=\theta_{a} \mid \theta_{a}^{\max }$. Put $k(\beta)=\beta^{-\alpha}(0<\alpha<2)$. If $\left|\theta_{\boldsymbol{B}}^{\max }\right|<\left(r_{\boldsymbol{B}}-k(\beta)\right)|V|$, then $\left|\theta_{\boldsymbol{B}}^{\text {rem }}\right|>16 / 25 k(\beta)|V|$ for every sufficiently large $\beta$.

Taking into account the fact that the plain figure with the area of $N$ which has the shortest boundary is the square with the breadth of $N^{1 / 2}$ for a given number $N>0$, we have the following estimate

$$
\begin{aligned}
\left|\partial \theta_{B}\right| & \geqq 4\left(r_{B}-k(\beta)^{1 / 2}|V|^{1 / 2}+\frac{4}{5} k(\beta)^{1 / 2}|V|^{1 / 2}\right. \\
& \geqq 4 r_{B}^{1 / 2}|V|^{1 / 2}+\frac{3}{5} k(\beta)^{1 / 2}|V|^{1 / 2}
\end{aligned}
$$

for every sufficiently large $\beta$.

From Lemma 7.6, we have $\left|\partial \theta_{D}\right|>4\left(r_{D}-r(\beta)\right)^{1 / 2}|V|^{1 / 2}$. Hence, 


$$
\begin{aligned}
|\Lambda| & \geqq\left|\partial \theta_{B}\right|+\left|\partial \theta_{D}\right| \\
& \geqq 4\left(r_{B}^{1 / 2}+r_{D}^{1 / 2}\right)|V|^{1 / 2}+\frac{2}{5} k(\beta)^{1 / 2}|V|^{1 / 2} \quad \text { a.w. } P_{N_{V^{\prime}}^{(1)}} \text {-prob. } 1 .
\end{aligned}
$$

On the other hand, from (7.14) we have

$$
|\Lambda|<4\left(r_{B}^{1 / 2}+r_{D}^{1 / 2}\right)|V|^{1 / 2}+k_{0}(\beta)|V|^{1 / 2} \quad \text { a.w. } P_{N_{V}^{(1)}} \text {-prob. } 1
$$

Because (7.24) is inconsistent with this, we have

$$
\left|\theta_{B}^{\max }\right|>\left(r_{B}-k(\beta)\right)|V| \text { a.w. } P_{N_{V}^{(1)}} \text {-prob. } 1 .
$$

By the same way, we have

$$
\left|\theta_{D}^{\max }\right|>\left(r_{D}-k(\beta)\right)|V| \quad \text { a.w. } P_{N_{V}^{(1)}} \text {-prob. } 1 .
$$

As $\theta_{B}^{\max }$ and $\theta_{D}^{\max }$ are connected subsets, the following estimates are easily obtained from (7.25) and (7.26),

$$
\left\{\begin{array}{l}
\left|\partial \theta_{B}^{\max }\right|>4 r_{B}^{1 / 2}|V|^{1 / 2}-k(\beta)|V|^{1 / 2} \\
\left|\partial \theta_{D}^{\max }\right|>4 r_{D}^{1 / 2}|V|^{1 / 2}-k(\beta)|V|^{1 / 2}
\end{array} \quad \text { a.w. } P_{N_{V}^{(1)}-\text { prob. } 1}\right.
$$

We shall prove

$$
\left|\partial \theta_{B}^{\max }\right|<4 r_{B}^{1 / 2}|V|^{1 / 2}+2 k_{0}(\beta)|V|^{1 / 2} \quad \text { a.w. } P_{N_{V}^{(1)}}^{(1)} \text { prob. } 1 .
$$

If $\left|\partial \theta_{B}^{\max }\right| \geqq 4 r_{B}^{1 / 2}|V|^{1 / 2}+2 k_{0}(\beta)|V|^{1 / 2}$, then from (7.27) we have

$$
|\Lambda|>4\left(r_{B}^{1 / 2}+r_{D}^{1 / 2}\right)|V|^{1 / 2}+k_{0}(\beta)|V|^{1 / 2} \quad \text { a.w. } P_{N_{V}^{(1)}} \text {-prob. } 1
$$

This is inconsistent with (7.14). Hence (7.28) is proved.

Next we shall prove Theorem 2.2. Let $r(\beta)$ be the function of $\beta$ satisfying $r(\beta) \sim \exp \left(-3 \beta \varepsilon_{1}\right)$.

In the similar way to the proof of Theorem 2.1, we have the following lemma.

Lemma 7.7. For sufficiently large $\beta$ and $V$, we have

$$
|| \theta_{a}\left|-r_{a}\right| V||<r(\beta)|V| \quad \text { a.w. } P_{N_{V}^{(2)}} \text {-prob. } 1(a=B \text { or } C) .
$$

Let $k(\beta)$ be the function of $\beta$ satisfying $k(\beta) \sim \exp \left(-2 \beta \varepsilon_{1}\right)$. If $\Gamma_{A, B}^{\max } \leqq$ $\left.4\left\{r_{B}+r_{C}\right)^{1 / 2}-k(\beta)\right\}|V|^{1 / 2}$, then

$$
\begin{aligned}
& |\Lambda| \geqq\left|\Gamma_{A, B}^{\max }\right|+\left|\Gamma_{B, C}\right|+\left|\Gamma_{C, D}\right|+\left|\Gamma_{D, A}\right| \\
& \geqq 4\left\{\left(r_{B}+r_{C}\right)^{1 / 2}-k(\beta)\right\}|V|^{1 / 2}+4\left(r_{C}-r(\beta)\right)^{1 / 2}|V|^{1 / 2} \\
& +4\left[r_{B}-r(\beta)-\left\{\left(\left(r_{B}+r_{C}\right)^{1 / 2}-k(\beta)\right)^{2}-\left(r_{C}-r(\beta)\right)\right\}\right]^{1 / 2}|V|^{1 / 2} \\
& \geqq 4\left\{\left(r_{B}+r_{C}\right)^{1 / 2}+r_{C}^{1 / 2}\right\}|V|^{1 / 2}+\left(r_{B}+r_{C}\right)^{1 / 4} k(\beta)^{1 / 2}|V|^{1 / 2} \text {. }
\end{aligned}
$$

This is inconsistent to the assertion of Proposition 7.2. Hence, we have 


$$
\Gamma_{A, B}^{\max } \geqq 4\left\{\left(r_{B}+r_{C}\right)^{1 / 2}-k(\beta)\right\}|V|^{1 / 2} \text { a.w. } P_{N_{V}^{(2)}} \text {-prob. } 1 .
$$

By the similar way we have

$$
\Gamma_{B, C}^{\max } \geqq 4\left(r_{C}^{1 / 2}-k(\beta)\right)|V|^{1 / 2} \quad \text { a.w. } P_{N_{V}^{(2)}} \text {-prob. } 1 .
$$

Hence the third and fourth assertions of Theorem 2.2 are proved. The fifth assertion is trivial.

\section{Appendix. Proof of Lemma 7.2}

Let $E^{\prime}(\cdot)$ be any function defined on $\Gamma$. We denote the Gibbs measure on $\Omega_{\theta, A}$ with respect to $E^{\prime}(\cdot)$ by $P_{\theta, A, E^{\prime}}$. Then we have the following for a given family of nonnegative integers $\{c(\bar{\gamma})\}_{\bar{\gamma}: c_{0}-i m a 11}$,

$$
\begin{aligned}
& P_{\theta, A, E^{\prime}}\left(N(\bar{\gamma}: \xi)=c(\bar{\gamma}) \quad \text { for all } c_{0} \text {-small } \bar{\gamma},\right. \\
& \left.\quad \text { and } N(\bar{\gamma} ; \xi)=0 \text { for all } c_{0} \text {-large } \bar{\gamma}\right) \\
& =\frac{1}{Z_{\theta, A, E^{\prime}}} N(\{c(\bar{\gamma})\} \mid \theta) \prod_{\bar{\gamma}: c 0^{-} \text {small }}\left(Z_{\theta(\gamma), i(\bar{\gamma}), E^{\prime}} \exp \left(-\beta E^{\prime}(\bar{\gamma})\right)\right)^{c(\bar{\gamma})}
\end{aligned}
$$

where $N(\{c(\bar{\gamma})\} \mid \theta)=\#\left\{\xi \in \Omega_{\theta, A} ; N(\bar{\gamma} ; \xi)=c(\bar{\gamma})\right.$ for all $c_{0}$-small $\bar{\gamma}$ and $N(\bar{\gamma} ; \xi)$ $=0$ for all $c_{0}$-large $\left.\bar{\gamma}\right\}$. From (A.1) we have

$$
N(\{c(\gamma)\} \mid \theta) \leqq Z_{\theta, A, E} \cdot \prod_{\bar{\gamma}: c_{0} \text {-small }}\left(e^{\beta E^{\prime}(\bar{\gamma})} / Z_{\theta(\gamma), i(\bar{\gamma}), E^{\prime}}\right)^{c(\bar{\gamma})} .
$$

When $E(\cdot)$ is the function on $\Gamma$ given in Section 2, we write $P_{\theta, A, E}=P_{\theta, A}$. From (A.1) and (A.2), we have

$$
\begin{aligned}
& P_{\theta, A}\left(N(\bar{\gamma} ; \xi)=c(\bar{\gamma}) \quad \text { for all } c_{0} \text {-small } \bar{\gamma}\right. \\
& \left.\quad \text { and } N(\bar{\gamma} ; \xi)=0 \text { for all } c_{0} \text {-large } \bar{\gamma}\right) \\
& \leqq \exp \left[\operatorname { i n f } _ { E ^ { \prime } } \left\{\ln Z_{\theta, A, E^{\prime}}-\ln Z_{\theta, A, E}-\beta \sum_{\bar{\gamma}: c_{0} \text {-small }} c(\bar{\gamma}) \Delta E(\bar{\gamma})\right.\right. \\
& \left.\left.\quad-\sum_{\bar{\gamma}: c_{0} \text {-small }} c(\bar{\gamma})\left(\ln Z_{\theta(\gamma), i(\bar{\gamma}), E^{\prime}}-\ln Z_{\theta(\gamma), i(\bar{\gamma}), E}\right)\right\}\right]
\end{aligned}
$$

where $\Delta E(\bar{\gamma})=E^{\prime}(\bar{\gamma})-E(\bar{\gamma})$.

We denote the terms in the bracket \{\} in (A.3) by $F_{\theta, A, E}\left(E^{\prime}\right)$. Then by using correlation functions $F_{\theta, A, E}\left(E^{\prime}\right)$ is expressed as follows,

$$
\begin{aligned}
& F_{\theta, A, E}\left(E^{\prime}\right) \\
& =\sum_{\bar{\gamma}}-\frac{\partial \ln Z_{\theta, A, E^{\prime}} \Delta E(\bar{\gamma})}{\partial E^{\prime}(\bar{\gamma})} \quad \\
& \quad+\left.\sum_{\bar{\gamma}_{1, \bar{\gamma}_{2}}} \frac{\partial^{2} \ln Z_{\theta, A, E^{\prime}}}{\partial E^{\prime}\left(\bar{\gamma}_{1}\right) \partial E^{\prime}\left(\bar{\gamma}_{2}\right)}\right|_{E^{\prime}=E+\alpha \Delta E} \Delta E\left(\bar{\gamma}_{1}\right) \Delta E\left(\bar{\gamma}_{2}\right) \quad(0<\alpha<1)
\end{aligned}
$$




$$
\begin{aligned}
& -\beta \sum_{\bar{\gamma} \in \Gamma_{A}^{c_{0}}} \Delta E(\bar{\gamma}) c(\bar{\gamma})-\sum_{\bar{\gamma} \in \Gamma_{A}^{c_{0}}} c(\bar{\gamma}) \sum_{\overline{\bar{\Delta}}} \frac{\partial \ln Z_{\theta(\gamma), i(\bar{\gamma}), E^{\prime}}}{\partial E^{\prime}(\bar{\Delta})} \Delta E(\bar{\Delta}) \\
& -\left.\sum_{\bar{\gamma} \in \Gamma_{A}^{c_{0}}} c(\bar{\gamma}) \sum_{\bar{\lambda}_{1} \bar{\lambda}_{2}} \frac{\partial^{2} \ln Z_{\theta(\gamma), i(\bar{\gamma}), E^{\prime}}}{\partial E^{\prime}\left(\bar{\lambda}_{1}\right) \partial E^{\prime}\left(\bar{\lambda}_{2}\right)}\right|_{E^{\prime}=E+\alpha \Delta E} \Delta E\left(\bar{\lambda}_{1}\right) \Delta E\left(\bar{\lambda}_{2}\right) \\
& =-\beta \sum_{\bar{\gamma}} \sum_{\substack{\bar{\Gamma} \in \bar{\gamma} \\
\Gamma \subset \theta}} \tau_{\theta, A, E^{\prime}}(\bar{\Gamma}) \Delta E(\bar{\gamma}) \\
& +\beta^{2} \sum_{\substack{\bar{\gamma}_{1} \neq \bar{\gamma}_{2} \\
\bar{\Gamma}_{1} \in \bar{\gamma}_{1, \bar{\Gamma}_{2} \in \bar{\gamma}_{2}}}}\left\{\tau_{\theta, A, E^{\prime}}\left(\bar{\Gamma}_{1}, \bar{\Gamma}_{2}\right)-\tau_{\theta, A, E^{\prime}}\left(\bar{\Gamma}_{1}\right) \tau_{\theta, A, E^{\prime}}\left(\bar{\Gamma}_{2}\right)\right\} \cdot \\
& \Delta E\left(\bar{\gamma}_{1}\right) \Delta E\left(\bar{\gamma}_{2}\right) \\
& +\beta^{2} \sum_{\bar{\gamma}} \sum_{\substack{\bar{\Gamma} \in \bar{\gamma} \\
\Gamma \subset \theta}}\left\{\tau_{\theta, A, E^{\prime}}(\bar{\Gamma})-\tau_{\theta, A, E^{\prime}}(\bar{\Gamma})^{2}\right\} \Delta E(\bar{\gamma})^{2} \\
& +\beta \sum_{\bar{\gamma} \in \Gamma_{A}^{c_{0}}} \Delta E(\bar{\gamma}) c(\bar{\gamma}) \\
& +\beta \sum_{\bar{\gamma} \in \Gamma_{\boldsymbol{A}}^{c_{0}}} c(\bar{\gamma}) \sum_{\bar{\Delta} \in \Gamma} \sum_{\bar{\Gamma} \in \bar{\Delta} \Gamma \subset \theta(\gamma)} \tau_{\theta(\gamma), i(\bar{\gamma}), E^{\prime}}(\bar{\Gamma}) \Delta E(\bar{\Delta}) \\
& -\sum_{\bar{\gamma} \in \Gamma_{A}^{c_{0}}} c(\bar{\gamma}) \sum_{\bar{\Delta}_{1}, \bar{\Delta}_{2} \in \Gamma} \frac{\partial^{2} \ln Z_{\theta(\gamma), i(\bar{\gamma}), E^{\prime}}}{\partial E^{\prime}\left(\bar{\Delta}_{1}\right) \partial E^{\prime}\left(\bar{\Delta}_{2}\right)} \Delta E\left(\bar{\Delta}_{1}\right) \Delta E\left(\bar{\Delta}_{2}\right) \\
& \equiv I_{1}+I_{2}+I_{3}+I_{4}+I_{5}+I_{6} \text {, }
\end{aligned}
$$

where $\Gamma_{A}=\{\bar{\gamma} \in \Gamma ; o(\bar{\gamma})=A\}, \quad \Gamma_{A}^{c_{0}}=\left\{\bar{\gamma} \in \Gamma_{A} ; \bar{\gamma}: c_{0}\right.$-small $\}$.

For any contour $\bar{\gamma} \in \Gamma_{A}^{c_{0}}$, put

$$
s(\bar{\gamma})=c(\bar{\gamma})-\sum_{\bar{\Gamma} \in \bar{\gamma}, \Gamma \subset \theta} \rho_{\theta, A}\left(\bar{\Gamma}, E^{\prime}\right) .
$$

Then $I_{1}+I_{4}+I_{5}$ is expressed as follows,

$$
\text { (A.6) } \begin{aligned}
I_{1}+ & I_{4}+I_{5} \\
= & -\beta \sum_{\bar{\Gamma} \in \Gamma_{\theta}} \tau_{\theta, A, E^{\prime}}(\bar{\Gamma}) \Delta E(\bar{\Gamma})+\beta \sum_{\bar{\gamma} \in \Gamma^{c_{0}}} c(\bar{\gamma})\{\Delta E(\bar{\gamma}) \\
& \left.+\sum_{\Gamma \subset \theta(\gamma)} \tau_{\theta,(\gamma), i(\bar{\gamma}), E^{\prime}}(\bar{\Gamma}) \Delta E(\bar{\Gamma})\right\} \\
= & -\beta \sum_{\bar{\Gamma} \in \Gamma_{A}} \rho_{\theta, A, E^{\prime}}(\bar{\Gamma}) \Delta E(\bar{\Gamma}) \\
& -\beta \sum_{\bar{\Gamma} \in \Gamma_{A}} \sum_{\bar{\Lambda}: A \supset \theta(\Gamma)} \rho_{\theta, A, E^{\prime}}(\bar{\Lambda}) \tau_{\theta(\Lambda), i(\bar{\Lambda}), E^{\prime}}(\bar{\Gamma}) \Delta E(\bar{\Gamma}) \\
& -\beta \sum_{\bar{\gamma} \in \Gamma_{A}^{c} \bar{\Lambda}: A \supset \theta(\Gamma)} \rho_{\theta, A, E^{\prime}}(\bar{\Lambda}) \tau_{\theta(\Lambda), i(\bar{\Lambda}), E^{\prime}}(\bar{\Gamma}) \Delta E(\bar{\Gamma}) \\
& +\beta \sum_{\bar{\gamma} \in \Gamma_{A}^{c_{0}}}\left(s(\bar{\gamma})+\sum_{\bar{\Gamma} \in \bar{\gamma}} \rho_{\theta, A, E^{\prime}}(\bar{\Gamma})\right) \Delta E(\bar{\gamma}) \\
& +\beta \sum_{\bar{\gamma} \in \Gamma_{A}^{c_{0}}}\left(s(\bar{\gamma})+\sum_{\bar{\Gamma} \in \bar{\gamma}} \rho_{\theta, A, E^{\prime}}(\bar{\Gamma})\right) \sum_{A \subset \theta(\gamma)} \tau_{\theta(\gamma), i(\bar{\gamma}), E^{\prime}}(\bar{\Lambda}) \Delta E(\bar{\Lambda})\left(\Gamma_{A}^{c}=\Gamma \backslash \Gamma_{A}\right) \\
= & -\beta \sum_{\bar{\Gamma} \in \Gamma_{A} \backslash \Gamma_{A}^{c_{0}}} \rho_{\theta, A, E^{\prime}}(\bar{\Gamma}) \Delta E(\bar{\Gamma})
\end{aligned}
$$




$$
\begin{aligned}
& -\beta \sum_{\bar{A} \in \Gamma_{A} \bar{\Gamma} \in \Gamma_{A}: \Gamma \subset \theta(\Lambda)} \rho_{\theta, A, E^{\prime}}(\bar{\Lambda}) \tau_{\theta(\Lambda), i(\bar{\Lambda}), E^{\prime}}(\bar{\Gamma}) \Delta E(\bar{\Gamma}) \\
& -\beta \sum_{\bar{\Lambda} \in \Gamma_{A} \bar{\Gamma} \in \Gamma_{A}^{c}: \bar{\Gamma} \subset(\Lambda \theta)} \rho_{\theta, A, E^{\prime}}(\bar{\Lambda}) \tau_{\theta(\Lambda), i(\bar{\Lambda}), E^{\prime}}(\bar{\Gamma}) \Delta E(\bar{\Gamma}) \\
& +\beta \sum_{\bar{\Gamma} \in \Gamma_{A}^{c_{0}}} \rho_{\theta, A, E^{\prime}}(\bar{\Gamma}) \sum_{\bar{\Lambda}: \Lambda \in \theta(\Gamma)} \tau_{\theta(\Gamma), i(\bar{\Gamma}), E^{\prime}}(\bar{\Lambda}) \Delta E(\bar{\Lambda}) \\
& +\beta \sum_{\bar{\gamma} \in \Gamma^{c_{0}}} s(\bar{\gamma})\left(\Delta E(\bar{\gamma})+\sum_{\bar{\Lambda}: \Lambda \subset \theta(\gamma)} \tau_{\theta(\gamma), i(\bar{\gamma}), E^{\prime}}(\bar{\Lambda}) \Delta E(\bar{\Lambda})\right) \\
& =-\beta \sum_{\bar{\Gamma} \in \Gamma_{A} \backslash \Gamma_{A}^{c_{0}}} \rho_{\theta, A, E^{\prime}}(\bar{\Gamma})\left\{\Delta E(\bar{\Gamma})+\sum_{\bar{A}: \Delta \subset \theta(\Gamma)} \tau_{\theta(\Gamma), i(\bar{\Gamma}), E^{\prime}}(\bar{\Lambda}) \Delta E(\bar{\Lambda})\right\} \\
& +\beta \sum_{\bar{\gamma} \in \Gamma_{A}^{c_{0}}} s(\bar{\gamma})\left\{\Delta E(\bar{\gamma})+\sum_{\bar{\Lambda}: \Lambda \subset \theta(\gamma)} \tau_{\theta(\gamma), i(\bar{\gamma}), E^{\prime}}(\bar{\Lambda}) \Delta E(\bar{\Lambda})\right\} .
\end{aligned}
$$

As $-I_{6}$ is the sum of variances of some quantities, $I_{6} \leqq 0$.

By using the clustering properties of correlation functions, we have (A.7) $\left|I_{2}\right|+\left|I_{3}\right|$

$$
\begin{aligned}
& \leqq \beta^{2} \sum_{\bar{\gamma}_{1} \neq \bar{\gamma}_{2}}\left\{\sum_{\substack{\bar{T}_{1} \in \bar{\gamma}_{1} \bar{\Gamma}_{2} \in \bar{\gamma}_{2} \\
\theta\left(\Gamma_{1}\right) \cap \theta\left(\Gamma_{2}\right)=\phi}} \mid \tau_{\theta, A, E^{\prime}}\left(\bar{\Gamma}_{1}, \bar{\Gamma}_{2}\right)\right. \\
& \left.-\tau_{\theta, A, E^{\prime}}\left(\bar{\Gamma}_{1}\right) \tau_{\theta, A, E^{\prime}}\left(\bar{\Gamma}_{2}\right)\left|\Delta E\left(\bar{\gamma}_{1}\right) \Delta E\right| \bar{\gamma}_{2}\right) \\
& +\sum_{\substack{\bar{\Gamma}_{1} \in \bar{\gamma}_{1}, \bar{\Gamma}_{2} \in \bar{\gamma}_{2} \\
\Gamma_{2} \subset \theta\left(\Gamma_{1}\right) \text { or } \Gamma_{1} \subset \theta\left(\Gamma_{2}\right)}} \mid \tau_{\theta, A, E^{\prime}}\left(\bar{\Gamma}_{1}, \bar{\Gamma}_{2}\right) \\
& \left.-\tau_{\theta, A, E^{\prime}}\left(\bar{\Gamma}_{1}\right) \tau_{\theta, A, E^{\prime}}\left(\bar{\Gamma}_{2}\right) \mid \Delta E\left(\bar{\gamma}_{1}\right) \Delta E\left(\bar{\gamma}_{2}\right)\right\} \\
& +\beta^{2} \sum_{\bar{\gamma}} \sum_{\bar{\Gamma} \in \bar{\gamma}, \Gamma \subset \theta} e^{-\beta E^{\prime}(\bar{\gamma})} \Delta E(\bar{\gamma})^{2} \\
& \leqq A_{1}(\beta) \cdot|\theta| \cdot \sum_{\bar{\gamma}_{1} \neq \bar{\gamma}_{2}: c_{0} \text {-small }} 2^{\left|\gamma_{1}\right|+\left|\gamma_{2}\right|} e^{-\beta E^{\prime}\left(\bar{\gamma}_{1}\right)-\beta E^{\prime}\left(\bar{\gamma}_{2}\right)} \cdot \Delta E\left(\bar{\gamma}_{1}\right) \cdot \Delta E\left(\bar{\gamma}_{2}\right) \\
& +2 \beta^{2} \cdot|\theta| \sum_{\bar{\gamma}_{1} \neq \bar{\gamma}_{2}: c_{0} \text {-small }}\left|\gamma_{1}\right|^{2}\left|\gamma_{2}\right|^{2} e^{-\beta E^{\prime}\left(\bar{\gamma}_{1}\right)-\beta E^{\prime}\left(\bar{\gamma}_{2}\right)} \Delta E\left(\bar{\gamma}_{1}\right) \Delta E\left(\bar{\gamma}_{2}\right) \\
& +\beta^{2}|\theta| \sum_{\bar{\gamma}: c 0^{- \text {small }}} e^{-\beta E^{\prime}(\bar{\gamma})} \Delta E(\bar{\gamma})^{2}
\end{aligned}
$$

where $A_{1}(\beta) \sim \beta^{2} \exp \left(-(3 / 4) \beta \varepsilon_{1}\right)$.

Hence,

$$
\begin{aligned}
& F_{\theta, A, E}\left(E^{\prime}\right) \leqq \beta^{2}|\theta|\left\{3 \sum_{\bar{\gamma}_{1} \neq \bar{\gamma}_{2}}\left|\gamma_{1}\right| \cdot\left|\gamma_{2}\right| 2^{\left|\gamma_{1}\right|+\left|\gamma_{2}\right|} e^{-\beta E^{\prime}\left(\bar{\gamma}_{1}\right)-\beta E^{\prime}\left(\bar{\gamma}_{2}\right)} \Delta E\left(\bar{\gamma}_{1}\right) \Delta E\left(\bar{\gamma}_{2}\right)\right. \\
& \left.+\sum_{\bar{\gamma}: c 0^{- \text {small }}} e^{-\beta E^{\prime}(\bar{\gamma})} \Delta E(\bar{\gamma})^{2}\right\} \\
& -\beta \sum_{\bar{\Gamma} \in \Gamma, \Gamma_{A}^{c_{0}}} \rho_{\theta, A, E^{\prime}}(\bar{\Gamma})\left\{\Delta E(\bar{\Gamma})+\sum_{\bar{\Lambda}: \Lambda \subset \theta(\Gamma)} \tau_{\theta(\Gamma), i(\bar{\Gamma}), E^{\prime}}(\bar{\Lambda}) \Delta E(\bar{\Lambda})\right\} \\
& +\beta \sum_{\bar{\gamma} \in \Gamma_{A}^{c_{0}}} s(\bar{\gamma})\left\{\Delta E(\bar{\gamma})+\sum_{\Gamma \subset \theta(\gamma)} \tau_{\theta(\gamma), i(\bar{\gamma}), E^{\prime}}(\bar{\Gamma}) \Delta E(\bar{\Gamma})\right\} .
\end{aligned}
$$

We take the following restriction on $E(\bar{\gamma})$ 
(A.9) $\left\{\begin{array}{l}\Delta E(\bar{\gamma})=-\frac{1}{4} \operatorname{sgn}(s(\bar{\gamma}))|\gamma| e^{\lambda|\gamma|}|V|^{-1 / 4} u \varepsilon_{1}, \bar{\gamma} \in \Gamma_{A}^{c_{0}} \quad\left(|\Delta E(\bar{\gamma})|<\frac{1}{4} \varepsilon_{1}|\gamma|\right) \\ \Delta E(\bar{\gamma})=0, \quad \bar{\gamma} \notin \Gamma_{A}^{c_{0}}\end{array}\right.$

where $0<u<|V|^{1 / 20}$. Put

$$
S_{\lambda}=\sum_{\bar{\gamma}: c 0^{-s m a l l}}|S(\bar{\gamma})||\gamma| e^{\lambda|\gamma|} .
$$

We estimate the right hand side of (A.8) under the condition (A.9). First we have

(A.11) |the sum of first and second terms in (A.8)|

$$
\begin{aligned}
\leqq & \frac{3}{16} \beta^{2}|\theta| \cdot \frac{u^{2}}{|V|^{1 / 2}}\left\{\sum_{\bar{\gamma}}|\gamma|^{2} 2|\gamma| e^{-\beta E^{\prime}(\bar{\gamma})+\lambda|\gamma|}\right\}^{2} \varepsilon_{1}^{2} \\
& +\frac{\beta^{2}}{16}|\theta| \cdot \frac{u^{2}}{|V|^{1 / 2}}\left\{\sum_{\bar{\gamma}}|\gamma|^{2} e^{-\beta E^{\prime}(\bar{\gamma})+2 \lambda|\gamma|}\right\} \cdot \varepsilon_{1}^{2} \\
& +\frac{A_{2}(\beta)|\theta|}{|V|^{(3 / 4) \beta c_{0}} \leqq A(\beta) \cdot|\theta| \cdot \frac{u^{2}}{|V|^{1 / 2}}}
\end{aligned}
$$

where $A(\beta) / \exp \left(-2 \beta \varepsilon_{1}\right) \downarrow 0$ exponentially as $\beta \rightarrow \infty$. The third term in (A.8) is estimated as follows,

the third term in (A.8)

$$
\begin{aligned}
\leqq & -\beta \sum_{\bar{\gamma} \in \Gamma_{A}^{c_{0}}}|s(\bar{\gamma})||\gamma| e^{\lambda|\gamma|} \cdot \frac{u}{|V|^{1 / 4}} \\
& +\beta \sum_{\bar{\gamma}, \bar{\gamma}^{\prime} \in \Gamma_{A}^{c_{0}}}|s(\bar{\gamma})||\gamma|^{2}\left|\gamma^{\prime}\right| e^{-\beta E^{\prime}\left(\bar{\gamma}^{\prime}\right)+\lambda\left|\gamma^{\prime}\right|} \cdot \frac{u}{|V|^{1 / 4}} \\
\leqq & -\beta s_{\lambda} \frac{u}{|V|^{1 / 4}}+\beta \operatorname{Max}_{k \geqq 4} k e^{-\lambda k} \cdot s_{\lambda} \cdot \frac{u}{|V|^{1 / 4}} \sum_{\bar{\gamma}^{\prime} \in \Gamma_{A}^{c_{0}}}\left|\gamma^{\prime}\right| e^{-\beta E^{\prime}\left(\bar{\gamma}^{\prime}\right)+\lambda\left|\gamma^{\prime}\right|} \\
= & -\beta s_{\lambda} \frac{u}{|V|^{1 / 4}}+B(\beta) \cdot s_{\lambda} \cdot \frac{u}{|V|^{1 / 4}}
\end{aligned}
$$

where $\beta A(\beta) \sim B(\beta)$. Hence, we have

the r.h.s. of $(\mathrm{A} .3) \leqq-\beta s_{\lambda} \frac{u}{|V|^{1 / 4}}+A(\beta) \cdot|\theta| \cdot \frac{u^{2}}{|V|^{1 / 2}}+B(\beta) \cdot s_{\lambda} \frac{u}{|V|^{1 / 4}} \cdot$

Put

$$
s_{\lambda}(\theta)=|\theta|^{-3 / 4} s_{\lambda}
$$

Hence, we have

(A.13) $P_{\theta, A}\left(N(\bar{\gamma} ; \xi)=c(\bar{\gamma})\right.$ for all $c_{0}$-small $\bar{\gamma}$ and $N(\bar{\gamma} ; \xi)=0$ for all $c_{0}$-large $\left.\bar{\gamma}\right)$

$$
\leqq \exp \left\{\inf _{0<u<|V|^{1 / 20}}\left[A(\beta)|\theta| \cdot \frac{u^{2}}{|V|^{1 / 2}}-(\beta-B(\beta)) s_{\lambda} \cdot \frac{u}{|V|^{1 / 4}}\right]\right\}
$$




$$
\begin{aligned}
= & \exp \left\{| \theta | ^ { 1 / 2 } \operatorname { i n f } _ { 0 < u < | V | ^ { 1 / 2 0 } } \left[A(\beta)\left(\frac{|\theta|^{1 / 4}}{|V|^{1 / 4}} u\right)^{2}\right.\right. \\
& \left.\left.-(\beta-B(\beta))\left(\frac{|\theta|^{1 / 4}}{|V|^{1 / 4}} u\right) s_{\lambda}(\theta)\right]\right\} \\
\leqq & \exp \left\{|\theta|^{1 / 2} \inf _{0<k^{1 / 4}|V|^{1 / 20}}\left[A(\beta) \eta^{2}-(\beta-B(\beta)) \eta s_{\lambda}(\theta)\right]\right\},
\end{aligned}
$$

and

$$
\begin{aligned}
& \quad \inf _{0<\eta<k^{1 / 4}|V|^{1 / 20}}\left[A(\beta) \eta^{2}-(\beta-B(\beta)) \eta s_{\lambda}(\theta)\right] \\
& \quad \leqq \begin{cases}-\left(\frac{\beta-B(\beta))^{2}}{4 A(\beta)} s_{\lambda}(\theta)^{2},\right. & \text { if } s_{\lambda}(\theta)<\frac{2 A(\beta)}{\beta-B(\beta)} k^{1 / 4}|V|^{1 / 20} \\
-A(\beta) k^{1 / 2}|V|^{1 / 10}, & \text { otherwise. }\end{cases}
\end{aligned}
$$

By using this estimate we have

(A.14) $P_{\theta, A}\left(u_{\lambda}(\xi ; \theta)>T\right.$ and $N(\bar{\gamma} ; \xi)=0$ for all $c_{0}$-large $\left.\bar{\gamma}\right)$

$$
<N_{c_{0}}\left[\exp \left\{-\frac{(\beta-B(\beta))^{2}}{4 A(\beta)} T^{2} \sqrt{|\theta|}\right\}+\exp \left(-A(\beta) k^{1 / 2}|V|^{1 / 10} \sqrt{|\theta|}\right)\right]
$$

where $N_{c_{0}}=\#\left\{\{c(\bar{\gamma})\} ; s_{\lambda}(\theta)>T\right\} . \quad$ By the standard argument, we have

$$
N_{c_{0}} \leqq \exp \left(24 \ln |V| \cdot|V|^{c_{0} \ln 3}\right) .
$$

As $c_{0} \ln 3<1 / 2$, we obtain the assertion of the lemma.

\section{Reference}

[1] Minlos, R. A. and Sinai, Ja. G., Math. Sbornik, 73 (1967), 115.

[2] - Trans. Moscow Math. Soc., 19 (1968), 121.

[ 3 ] Peierls, R. E., Proc. Cambridge Philos. Soc., 32 (1936), 477.

[4] Dobrushin, R. L., Functional Anal. Appl., 8 (1968), 302.

[5] Minlos, R., Russian Math. Surveys, 23 (1968), 137.

[6] Ruelle, D., Statistical Mechanics, Rigorous results, Benjamin, New York, 1969.

[7] Miyamoto, M., Phase transitions in lattice models, Seminar on Prob., 1972 (in Japanese).

[8] Gallavotti, G. and Miracle-Sole, S., Commun. math. Phys., 27 (1972), 103.

[9] Gertsik, V. M. and Dobrushin, R. L., Funkts. Analiz., \& (1972), 12. 
